\title{
Complement inhibitor CSMD1 modulates epidermal growth factor receptor oncogenic signaling and sensitizes breast cancer cells to chemotherapy
}

Chrysostomi Gialeli 1,2, Emre Can Tuysuz¹, Johan Staaf ${ }^{3}$, Safia Guleed', Veronika Paciorek , Matthias Mörgelin", Konstantinos S. Papadakos ${ }^{1}$ and Anna M. Blom ${ }^{1 *}$

\begin{abstract}
Background: Human CUB and Sushi multiple domains 1 (CSMD1) is a large membrane-bound tumor suppressor in breast cancer. The current study aimed to elucidate the molecular mechanism underlying the effect of CSMD1 in highly invasive triple negative breast cancer (TNBC).

Methods: We examined the antitumor action of CSMD1 in three TNBC cell lines overexpressing CSMD1, MDAMB-231, BT-20 and MDA-MB-486, in vitro using scanning electron microscopy, proteome array, GRT-PCR, immunoblotting, proximity ligation assay, ELISA, co-immunoprecipitation, immunofluorescence, tumorsphere formation assays and flow cytometric analysis. The mRNA expression pattern and clinical relevance of CSMD1 were evaluated in 3520 breast cancers from a modern population-based cohort.

Results: CSMD1-expressing cells had distinct morphology, with reduced deposition of extracellular matrix components. We found altered expression of several cancer-related molecules, as well as diminished expression of signaling receptors including Epidermal Growth Factor Receptor (EGFR), in CSMD1-expressing cells compared to control cells. A direct interaction of CSMD1 and EGFR was identified, with the EGF-EGFR induced signaling cascade impeded in the presence of CSMD1. Accordingly, we detected increased ubiquitination levels of EGFR upon activation in CSMD1-expressing cells, as well as increased degradation kinetics and chemosensitivity. Accordingly, CSMD1 expression rendered tumorspheres pretreated with gefitinib more sensitive to chemotherapy. In addition, higher mRNA levels of CSMD1 tend to be associated with better outcome of triple negative breast cancer patients treated with chemotherapy.

Conclusions: Our results indicate that CSMD1 cross-talks with the EGFR endosomal trafficking cascade in a way that renders highly invasive breast cancer cells sensitive to chemotherapy. Our study unravels one possible underlying molecular mechanism of CSMD1 tumor suppressor function and may provide novel avenues for design of better treatment.
\end{abstract}

Keywords: CSMD1, EGFR trafficking, Chemosensitivity, Breast cancer

${ }^{*}$ Correspondence: anna.blom@med.lu.se

${ }^{1}$ Department of Translational Medicine, Lund University, Malmö, Sweden

Full list of author information is available at the end of the article

\section{Background}

Human CUB and Sushi Multiple Domains 1 (CSMD1) is a large $(390 \mathrm{kDa})$ transmembrane complement inhibitor, highly expressed in testis and brain, but also present original author(s) and the source, provide a link to the Creative Commons licence, and indicate if changes were made. The images or other third party material in this article are included in the article's Creative Commons licence, unless indicated otherwise in a credit line to the material. If material is not included in the article's Creative Commons licence and your intended use is not permitted by statutory regulation or exceeds the permitted use, you will need to obtain permission directly from the copyright holder. To view a copy of this licence, visit http://creativecommons.org/licenses/by/4.0/. The Creative Commons Public Domain Dedication waiver (http://creativeco mmons.org/publicdomain/zero/1.0/) applies to the data made available in this article, unless otherwise stated in a credit line to the data. 
in lung, colon, thyroid gland, breast, and pancreas [1-3]. The large, extracellular portion of the protein consists of $14 \mathrm{~N}$-terminal CUB domains, interspaced by single complement control protein $(\mathrm{CCP})$ domains and a subsequent tandem array of $15 \mathrm{CCP}$ domains. The highly conserved C-terminal part is composed of a single transmembrane region followed by a short cytoplasmic tail of 56 amino acids accommodating several predicted phosphorylation sites on serine, threonine and tyrosine residues. CSMD1 is therefore a potential receptor/co-receptor with downstream signaling $[4,5]$ but no physiological ligands triggering its signaling have to date been identified.

The CSMD1 chromosomal locus spans over 2 million base pairs at the short arm of chromosome 8 (8p23.1) [4, 6]. Using genome-wide association studies, CSMD1 has been associated with several pathological processes, from neurodegenerative and psychiatric disorders to infertility and cancer [7-9]. For several years, CSMD1 has been proposed to act as a tumor suppressor since allelic loss, mutations, and methylations in its genomic region have been reported in several malignancies, including breast cancer [6, 10-15]. In addition, a decrease in CSMD1 expression has been linked to poor prognosis and shorter survival of cancer patients [14, 16-18]. However, there is only a handful of reports experimentally confirming the tumor suppressing properties of CSMD1 [18-23]. Thus, abrogation of CSMD1 expression in normal mammary human cells disrupts normal breast cell function and transformation, rendering the cells highly proliferative, migratory and invasive [22]. Forced expression of CSMD1 in human BT-20 and MDA-MB-231 triple negative breast cancer (TNBC) cells significantly inhibited their migration, adhesion and invasion, while stable silencing of CSMD1 expression in hormone-dependent T47D cells enhanced their migratory, adherent and clonogenic abilities. Moreover, expression of CSMD1 in the highly invasive MDA-MB-231 cells diminished their overall signaling potential and stem cell-like properties. Further, in a xenograft model, expression of CSMD1 entirely blocked the ability of MDA-MB-231 cells to metastasize in vivo, likely via inhibiting local invasion, but not extravasation into distant tissues [18]. However, the molecular mechanisms responsible for the suppressor effects of CSMD1 are unknown, and the current study therefore aimed to identify them.

\section{Methods}

\section{Cell lines, culture conditions and mammary tissues}

The human breast cancer cell lines MDA-MB-231, BT-20 and MDA-MB-468 were purchased directly from ATCC and all the experiments were performed on low passage cultures. Cells stably transfected to express CSMD1 were cultured with addition of $3 \mu \mathrm{g} / \mathrm{ml}$ puromycin
(Invitrogen). The generation of the stable transfectants has been described previously [18]. All cells were maintained in DMEM (HyClone) supplemented with $10 \%$ fetal bovine serum (FBS) and penicillin/streptomycin. All cells were tested for Mycoplasma contamination monthly. Throughout the manuscript CSMD1-expressing cells are denoted as CSMD1, while those containing the control empty-GFP vector were coded as CTRL. Cells were starved in serum-free medium for $2 \mathrm{~h}$ prior to all stimulation experiments. All stimulations were carried out in starvation medium for the indicated times.

\section{Scanning electron microscopy}

Cells were seeded on plastic cover slips and after a $24 \mathrm{~h}$ culture period were further processed as described previously [24].

\section{Proteome profiling}

A Proteome Profiler ${ }^{\mathrm{TM}}$ Human XL Oncology Array kit (R\&D, ARY026) was used to determine the relative levels of 84 human-cancer-related proteins according to manufacturer's instructions. CTRL and CSMD1-expressing MDA-MB-231 breast cancer cells were serum starved for $30 \mathrm{~min}$ to synchronize them and then lysed in the provided lysis buffer (300 $\mu \mathrm{g}$ protein/array). Cell culture supernatants were collected in Optimem serum free medium (Gibco) for $48 \mathrm{~h}$ and subsequently concentrated five times using Amicon filters (Millipore, $2 \mathrm{kDa}$ cutoff). Densities of individual dots corresponding to each protein were measured by the Image J software to compare CTRL and CSMD1-expressing cells.

\section{Real time PCR}

RNA was purified using RNAeasy Plus Mini kits (Qiagen) from tumors formed in vivo after orthotopic injection of MDA-MB-231 CTRL and CSMD1 cells into the mammary fat pad of 8 weeks old SCID (CB-17/Icr-Prk$\mathrm{dcscid} / \mathrm{Rj}$ ) mice as described [18], or from MDA-MB-231 CTRL and CSMD1 cells grown in vitro. Next, mRNA was reverse transcribed to cDNA with SuperScript III Reverse Transcriptase and gene expression was quantified using TaqMan Gene Expression Assays. mRNA expression levels were calculated using the $\triangle \mathrm{CT}$ method after normalization with the geometric mean of the three housekeeping genes; cyclophilin A (Hs99999904_m1), TATA box binding protein (Hs0042761_m1) and hypoxanthine phosphoribosyltransferase 1 (Hs99999909_ $\mathrm{m} 1)$. Genes assayed and their corresponding TaqMan Gene Expression Assays from ThermoFisher were: EGFR (Hs01076090m1), CTSS (Hs00175407m1), EGF (Hs01099990m1), TGF $\alpha$ (Hs00608187), and AREG (Hs00950669m1). 


\section{Flow cytometry}

Cells were seeded in 6-well plates and once $70 \%$ confluency was reached, detached with Versene (EDTA based buffer, Invitrogen), washed with FACS buffer $(10 \mathrm{mM}$ HEPES, $140 \mathrm{mM} \mathrm{NaCl}, 5 \mathrm{mM} \mathrm{KCl}, 1 \mathrm{mM} \mathrm{MgCl}, 2 \mathrm{mM}$ $\mathrm{CaCl}_{2}, 0.02 \% \mathrm{NaN}_{3}$ ), and incubated for $1 \mathrm{~h}$ with the appropriate antibody in FACS buffer at room temperature. After washing with FACS buffer cells were analyzed using Cytoflex flow cytometer (Beckman) and FlowJo software (BD). For discrimination between live, apoptotic, or necrotic cells, cells were stained with Annexin V-APC (Immunotools) and live/dead cell discrimination reagent Zombie Aqua (Biolegend) in FACS buffer for $30 \mathrm{~min}$, and then analyzed as above.

\section{Immunoprecipitation \& Immunodetection of proteins}

Total protein lysates were obtained with RIPA buffer (150 mM NaCl, $10 \mathrm{mM}$ Tris- $\mathrm{HCl}$ [pH 7.2], 0.1\% SDS, $1 \%$ Triton X-100) or NP40 buffer ( $150 \mathrm{mM} \mathrm{NaCl}, 50 \mathrm{mM}$ Tris- $\mathrm{HCl}$ [pH 7.5], 1\% NP40) containing a protease and phosphatase inhibitor cocktail (Thermo Fisher Scientific). When ubiquitination experiments were performed, lysates were also supplemented with DUBs inhibitor N-Ethylmaleimide (NEM). Protein concentrations in lysates were estimated with Pierce BCA Protein Assay Kit, equal amounts were separated by SDS-PAGE, and transferred onto PVDF membranes with a Trans-Blot Turbo Transfer Pack (Bio-Rad). For immunoprecipitation, $1 \mathrm{mg}$ of total protein was used with $50 \mu \mathrm{l}$ of Protein G or Protein A Dynabeads pre-coated with $5 \mu \mathrm{g}$ of capturing antibody. After overnight incubation, beads were extensively washed with NP40 buffer, and immunoprecipitated proteins were eluted with $30 \mu \mathrm{l}$ of Laemmli buffer. Primary and secondary antibodies used are listed in Table S1.

\section{Transfection}

Transfection of DNA constructs was performed using Lipofectamine 2000 (Invitrogen) following the manufacturer's instructions. $1 \mu \mathrm{g}$ of DNA was used for each transfection. pcDNA6A-EGFR ECD (1-644) (Addgene plasmid \#42,666; http://n2t.net/addgene: 42666; RRID:Addgene_42666) and pcDNA6A-EGFR ICD (645-1186) (Addgene plasmid \# 42,667; http:// n2t.net/addgene:42667; RRID:Addgene_42667) were a gift from Dr Mien-Chie Hung [25].

\section{Immunofluorescent \& dual link assay}

Imaging chamber slides from Ibidi were coated with Poly-D-lysine (Sigma), before cells seeding. The following day, cells were fixed with $4 \%$ formaldehyde (Merck) and permeabilized with $0.1 \%$ Triton X-100
(Merck). Proximity ligation assays was performed following the manufacturer's protocol for the Dual link kit (Sigma) using the appropriate antibodies (Table S1). For the immunofluorescent studies, cells were fixed as described above, blocked with 3\% BSA in PBS for $1 \mathrm{~h}$ at room temperature, and incubated with primary antibodies overnight at $4{ }^{\circ} \mathrm{C}$, followed by $1 \mathrm{~h}$ incubation with the corresponding Alexa-conjugated secondary antibodies at room temperature. Cells were washed and mounted using Prolong Glass medium (ThermoFisher Scientific) and visualized with ZEIS LSM800 confocal microscope. Nuclei were stained with DAPI or SYTOX Orange. Corresponding IgG controls were used as negative controls for each antibody staining. Co-localization quantification was performed using Manders' coefficient in Coloc2 plugin of FIJI software. The Manders M1 coefficient was expressed as a percentage (e.g. $\mathrm{M} 1=0.2$ was expressed as $20 \%$ ) to show the fraction of intensities in channel 1 that is colocalized with intensities in channel 2.

\section{EGFR degradation}

Cells were seeded and incubated overnight. Next day, cells were pre-treated with $100 \mu \mathrm{M}$ of cycloheximide (Sigma Aldrich) for $2 \mathrm{~h}$ and stimulated with EGF (Sigma Aldrich) in the presence of cycloheximide for indicated time points. Cells incubated with only cycloheximide were used to determine the initial amount of EGFR for each clone. $\mathrm{NH}_{4} \mathrm{Cl}(10 \mathrm{nM})$ and MG132 $(5 \mu \mathrm{M})$ were used to evaluate lysosome and proteasome contributions in EGFR degradation, respectively. EGFR recovery was assessed by calculating the ratio $\left[\mathrm{Cyclo}+\mathrm{NH}_{4} \mathrm{Cl}\right.$ (or MG132)+EGF] / [Cyclo+EGF].

\section{Detection of EGFR Dimers}

EGFR dimers were detected as described [26]. Briefly, cells were stimulated with EGF $(25 \mathrm{ng} / \mathrm{ml})$ and/or Gefitinib (GEF; $10 \mu \mathrm{M}$, Selleckchem) for $15 \mathrm{~min}$ at $37^{\circ} \mathrm{C}$. Following stimulation, cell surface proteins were crosslinked with $1.1 \mathrm{mM}$ of bis[sulfosuccinimidyl] suberate $\left(\mathrm{BS}^{3}\right.$, Thermo Scientific) for $30 \mathrm{~min}$ at room temperature. Cells were lysed and equal amounts of protein were separated by $3-8 \%$ Criterion $^{\mathrm{TM}}$ XT Tris-Acetate Protein Gel (Biorad), transferred onto PVDF membranes with TransBlot Turbo Transfer Pack and probed for EGFR using specific antibodies.

\section{Tumorsphere formation assay}

Cells at $1.5 \times 10^{5}$ (for MDA-MB-231) or $1 \times 10^{5}$ (for BT-20) per well were seeded onto AggreWell ${ }^{\text {TM }} 400$ plates (Stem Cell) and maintained in MammoC$\mathrm{ult}^{\mathrm{TM}}$ Human Medium Kit (Stem Cell) according to 
manufacturer's instructions to develop uniform tumorpheroids with consistent size and shape. Next day, medium was replaced with fresh medium containing $35 \mu \mathrm{M}$ of GEF followed by $2 \mu \mathrm{M}$ of doxorubicin or epirubicin treatment for two days (MDA-MB-231) or $8 \mu \mathrm{M}$ of doxorubicin or epirubicin treatment for one day (BT-20).

\section{Gene expression analysis of breast cancer tumors}

Gene expression profiles for 3520 primary breast cancer patients from the Swedish Cancerome Analysis Network - Breast (SCAN-B) study were obtained from the study by Vallon-Christersson et al., as processed fragments per kilobase per million reads (FPKM) RNA sequencing data, together with patient-matched clinical data. The cohort is population representative and patients were enrolled between 2010-2015 in South Sweden (PMID:31,434,940) [27].

\section{Statistical analysis}

All experiments were repeated at least 3 times with bars indicating mean \pm SD with black and grey circles indicating independent data points in CTRL and CSMD1 groups, respectively. The statistical analyses were performed using GraphPad Prism. The significance level was set at $\mathrm{p}<0.05$. Details about statistical methods are provided in figure legends. Survival analyses were performed in $\mathrm{R}$ version 3.6.1 using the survival package with overall survival (OS) or invasive disease-free survival (IDFS) as clinical endpoints. Survival curves were compared using Kaplan-Meier estimates and the log-rank test. Hazard ratios were calculated through univariable or multivariable Cox regression using the coxph $\mathrm{R}$ function. In multivariable analyses tumor size (mm), lymph node (LN) status (node-positive / node-negative), and tumor grade were included as covariates. The full available follow-up time was used in calculations.

\section{Results \\ Distinct proteomic signature of CSMD1-expressing MDA-MB-231 breast cancer cells}

In our previous study, we showed that CSMD1 expression in breast cancer cells (BCCs) decreased their metastatic potential [18]. When analyzed using scanning electron microscopy, MDA-MB-231 wild type (WT) and CTRL cells had a smoother surface, whereas MDA-MB-231 CSMD1 cells produced larger amounts of extracellular material and their surface contained a lot of globular vesicles. In addition, CSMD1-expressing cells were smaller and exhibited diminished formation of filopodia (cytoplasmic protrusions). Further, we only observed cell footprints indicating movement in WT and CTRL cells in contrast to the CSMD1 "amotile" cells (Figs. 1A-C). We hypothesized that this distinct morphological architecture of CSMD1-expressing cells is a result of altered cellular protein expression and remodeling, characteristic for oncogenic transformation and progression of cancer. Therefore, total protein cell extracts from MDAMB-231 CTRL and CSMD1-expressing cells (Figs. 1D-E), as well as their corresponding secretomes (supernatants) (Figs. 1F-G), were analyzed using Human Proteome Oncology Arrays. Overall, the results indicated several possible mediators of CSMD1 action, involving growth factor receptors and their ligands (EGFR/AREG, PDGFAA, VEGF), cysteine proteases (CTSS and CTSB), as well as adhesion and actin remodeling molecules (EpCAM, CapG) (Figs. 1E-G). Cathepsin S (CTSS) showed the greatest decrease in expression in MDA-MB-231 CSMD1 cells, followed by Epidermal Growth Factor Receptor (EGFR) and its ligand amphiregulin (AREG), as compared with CTRL cells. Consistent with our observations of altered morphology of the MDA-MB-231 CSMD1 cells, adhesion and actin remodeling molecules in the

\footnotetext{
(See figure on next page.)

Fig. 1 Distinct proteomic signature of CSMD1-expressing MDA-MB-231 BCCs. Scanning electron microscope images of MDA-MB-231 (A) WT, (B) CTRL and (C) CSMD1 BCCs showing distinct morphology of CSMD1-expressing cells. Large panels scale $100 \mu \mathrm{M}$. Small panels scale $20 \mu \mathrm{M}$. Black arrows indicate the cell "footprints". White arrows indicate the cytoplasmic protrusions. Dark grey arrows indicate the extracellular material-globular vesicles. Proteome Oncology profiler array —Cancer-related protein analysis of CTRL and CSMD1-expressing MDA-MB-231 cells. D, E Blots showing the location of proteins and capture antibodies spotted onto the array in duplicates. Positive and negative controls are indicated by + and adjacent to appropriate spots. Quantification of mean spot pixel intensities of CTRL and CSMD1 cells was plotted in the same order as spotted in the array when analyzing (D) cell lysates with the $(\mathbf{E})$ corresponding quantifications and $(\mathbf{F})$ cell culture supernatants with the $(\mathbf{G})$ corresponding quantifications. Numbers correspond to interesting findings in this array. Confirmation of the array: CTSS and EGFR expression in different CTRL and CSMD1 clones of MDA-MB-231 BCCs. Western blot analysis of total cell (H) lysates and (I) supernatants immunodetection of CTSS with $\beta$-actin used as a loading control, and (J) mRNA expression levels of CTSS. (L) Western blot analysis of total cell lysates immunodetecting EGFR and CSMD1 with $\beta$-tubulin used as a loading control, (M) cell surface EGFR expression assessed by flow cytometry, and (N) mRNA expression levels of EGFR. Shown is also mRNA expression of (K) CTSS and (O) EGFR in tumors formed in SCID mice injected with MDA-MB-231 CTRL and CSMD1 cells (5 mice in each group). All experiments were repeated at least 3 times. Bars indicate means \pm SD. One-way ANOVA Turkey's multiple comparisons test was used when comparing CSMD1 clones to CTRL clones, and Mann-Whitney comparison test was used when comparing CTRL and CSMD1 groups in tumors formed in vivo $\left({ }^{*}<0.05,{ }^{* *}<0.01,{ }^{* *}<0.001\right)$
} 

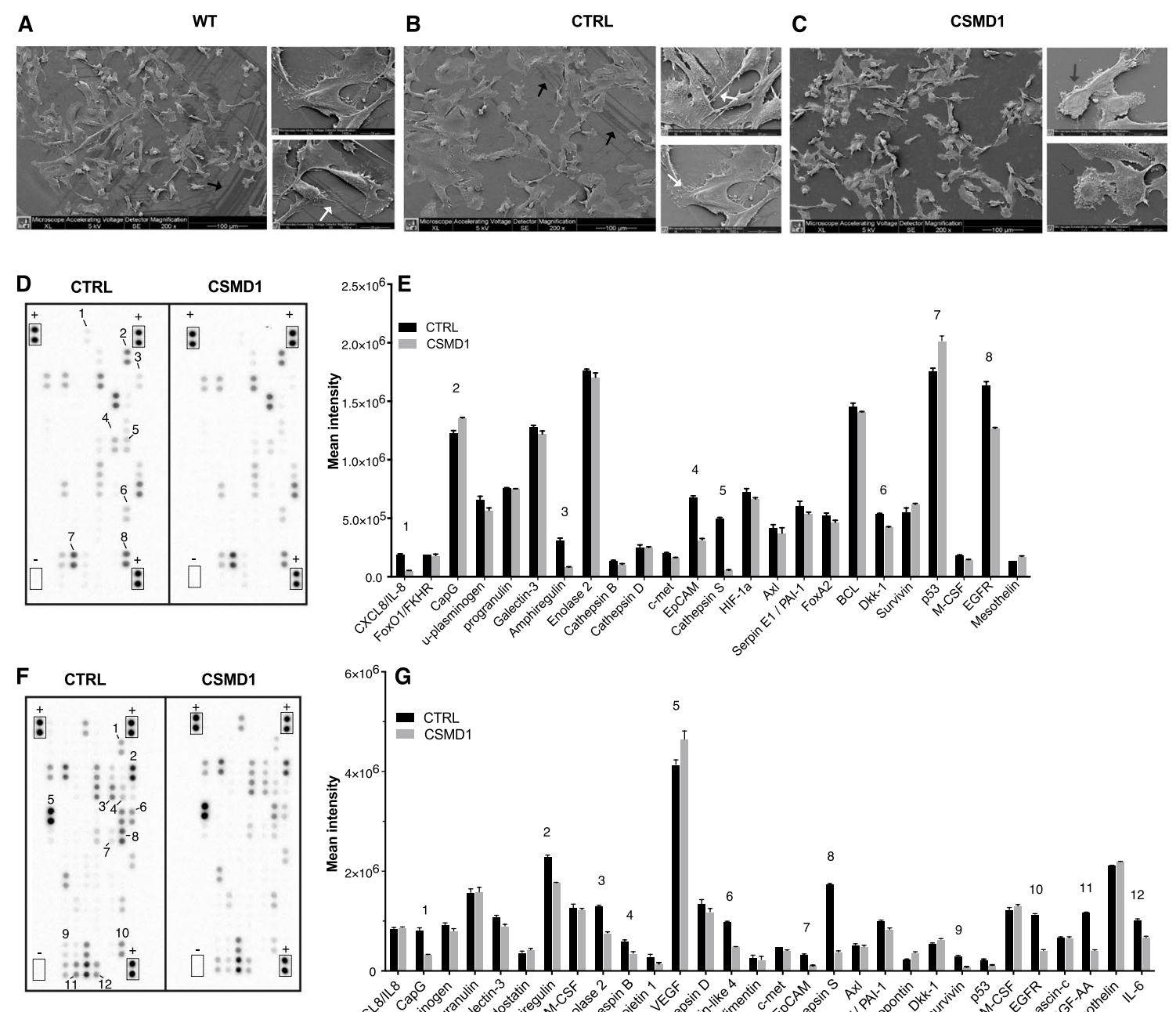

ve
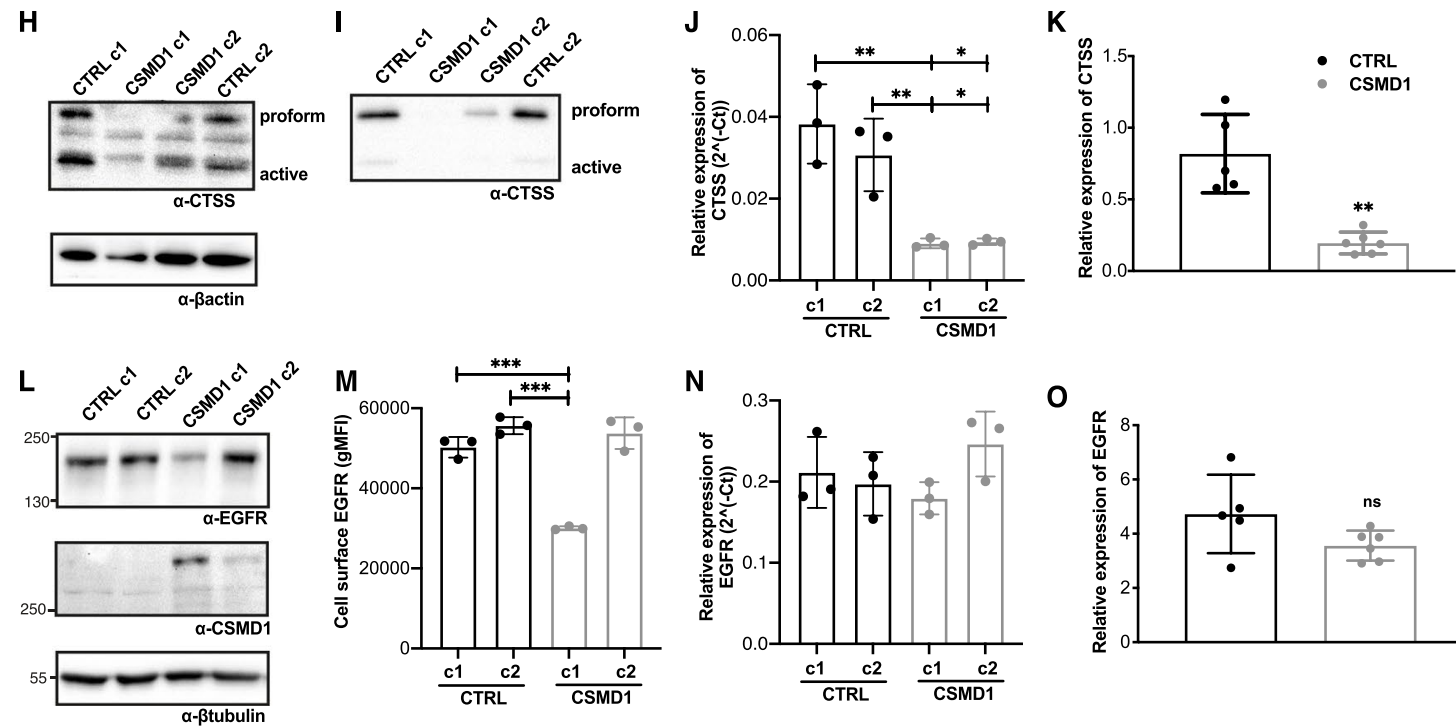

0

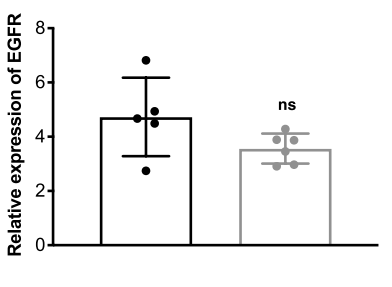

Fig. 1 (See legend on previous page.) 
array (EpCAM, CapG) were downregulated in CSMD1expressing cells. Other proteins, which also showed altered expression included proinflammatory cytokines, interleukins IL-8 and IL- 6 . On the other hand, the expression of p53 tumor suppressor was upregulated in MDA-MB-231 CSMD1 cells, compared to CTRL.

The protein array data were confirmed using different experimental approaches. First, CTSS expression was assessed in lysates (Fig. 1H) and corresponding supernatants (Fig. 1I) of several clones of CSMD1-expressing MDA-MB-231 cells, using Western blotting. CTSS expression and secretion from CSMD1-expressing cells was downregulated. Accordingly, CTSS mRNA levels were also significantly suppressed in MDA-MB-231 CSMD1 in comparison with CTRL cells (Fig. 1J), and this observation was confirmed in tumors formed in SCID mice injected with MDA-MB-231 CTRL and CSMD1 cells (Fig. 1K).

In addition, EGFR protein expression was assessed in lysates of several clones of CSDM1-expressing MDAMB-231 cells using Western blotting. We found that EGFR expression was negatively correlated with CSMD1 expression in the different clones. The clone $\mathrm{cl}$, highly expressing CSMD1, exhibited the lowest expression of EGFR, while the other CSMD1 clone expressing lower levels of CSMD1 exhibited comparable EGFR expression with the CTRL clones (Fig. 1L). We further confirmed this observation detecting cell-surface EGFR using flow cytometry (Fig. 1M), while the levels of mRNA coding for $E G F R$ (Fig. $1 \mathrm{~N}$ ), and its ligands such as $E G F, T G F \alpha$ and AREG were not altered in the CSMD1 clones in comparison with the CTRL cells (Supplementary Fig. 1A-C). Of note, as we have seen previously, the mRNA levels of CSMD1 seem to correlate with its protein expression in the different clones [28]. Accordingly, there was no difference in EGFR mRNA expression in tumors formed in SCID mice injected with MDA-MB-231 CTRL and CSMD1 cells (Fig. 1O). Further, mRNA expression databases for breast cancer patients available online, also did not indicate correlation between mRNA levels of EGFR and CSMD1 (Supplementary Fig. 1D \& E). Therefore, the observed difference on the protein level may depend on post-translational mechanisms such as receptor trafficking or degradation.

\section{CSMD1 interacts directly with EGFR}

EGFR (also known as ERBB or HER1) is a member of the HER cell-surface receptor tyrosine kinase family. Considering the localization of CSMD1 on the cell surface, we hypothesized that CSMD1 may act as a regulator of EGFR function. Indeed, a proximity ligation assay (PLA) indicated that CSMD1 interacted with EGFR in the MDA-MB-231 CSMD1 cells (Fig. 2A\&B). Furthermore, lysates of CTRL and CSMD1 MDAMB-231 cells were prepared in minimally denaturing conditions and analyzed by co-immunoprecipitation. CSMD1 was specifically detected in EGFR-precipitates by Western blotting, whereas no signal was observed either in the negative control (CTRL MDA-MB-231), or when isotype control antibodies were used (Fig. 2C). Furthermore, in immunoprecipitated CSMD1-complexes we detected a weaker signal approximately corresponding to the size of EGFR monomer $(175 \mathrm{kDa})$ and a stronger signal of EGFR dimers, while no such signals were detected with the corresponding isotype control antibodies (Fig. 2D). The direct binding between CSMD1 and EGFR was further confirmed using ELISA in which complexes were captured with immobilized anti-EGFR antibodies and detected with anti-CSMD1 antibodies (Fig. 2E). The EGFR-CSMD1 interaction was detected by ELISA in two different clones with varying overexpression levels of CSMD1. Furthermore, CTRL and CSMD1-expressing cells were transiently transfected with the Myc-tagged extracellular domain of EGFR (ECD) and Myc-tagged intracellular domain of EGFR (ICD). The efficient transfection

\footnotetext{
(See figure on next page.)

Fig. 2 CSMD1 interacts with EGFR. A Representative confocal z-stacked maximum projection images of PLA using MDA-MB-231 CTRL and CSMD1 cells. B Quantification of the dots per cell showing number of interactions between CSMD1 and EGFR. Binding between CSMD1 and EGFR was confirmed using co-immunoprecipitation (C) Cell lysates were immunoprecipitated using antibodies against EGFR and corresponding lgG control followed by immunodetection of CSMD1 protein. D Cell lysates were immunoprecipitated using antibodies against CSMD1 and corresponding IgG control followed by immunodetection of EGFR protein. E In ELISA setup an anti-EGFR antibody was used to capture the EGFR protein complexes lysed under non-denatured conditions and an anti-CSMD1 antibody applied to detect the EGFR-CSMD1 interactions. F MDA-MB-231 cell expressing CSMD1 and the control cells were transiently transfected with plasmid constructs that express extracellular domain of EGFR (ECD) and intracellular domain of EGFR (ICD) tagged with c-Myc. Representative blot detecting c-myc and confirming efficient transfection of the cells is shown. G The anti-c-myc capturing antibody was immobilized in ELISA plates, incubated with cell lysates followed by an anti-CSMD1 antibody to detect the myc-ECD-EGFR-CSMD1 and myc-ICD-EGFR-CSMD1 complexes. H Colocalization of CSMD1 and EGFR. MDA-MB-231 CSMD1-expressing cells (c1 clone) were fixed and stained with anti-EGFR (red) and anti-CSMD1 (green). DAPI (blue) was used to stain the nuclei. Scale bars $10 \mu \mathrm{m}$. All experiments were repeated at least 3 times with bars indicating mean \pm SD, black and grey circles correspond to independent data points for CTRL and CSMD1 groups, respectively. Unpaired t-test was used when comparing 2 samples, one-way ANOVA Dunnett's multiple comparisons test was used when compared 3 or more samples, and two-way ANOVA Bonferroni's multiple comparisons test was used when comparing 3 or more groups with 2 variables $\left.{ }^{*}<0.05,{ }^{* *}<0.01,{ }^{* * *}<0.001,{ }^{* * *}<0.0001\right)$
} 

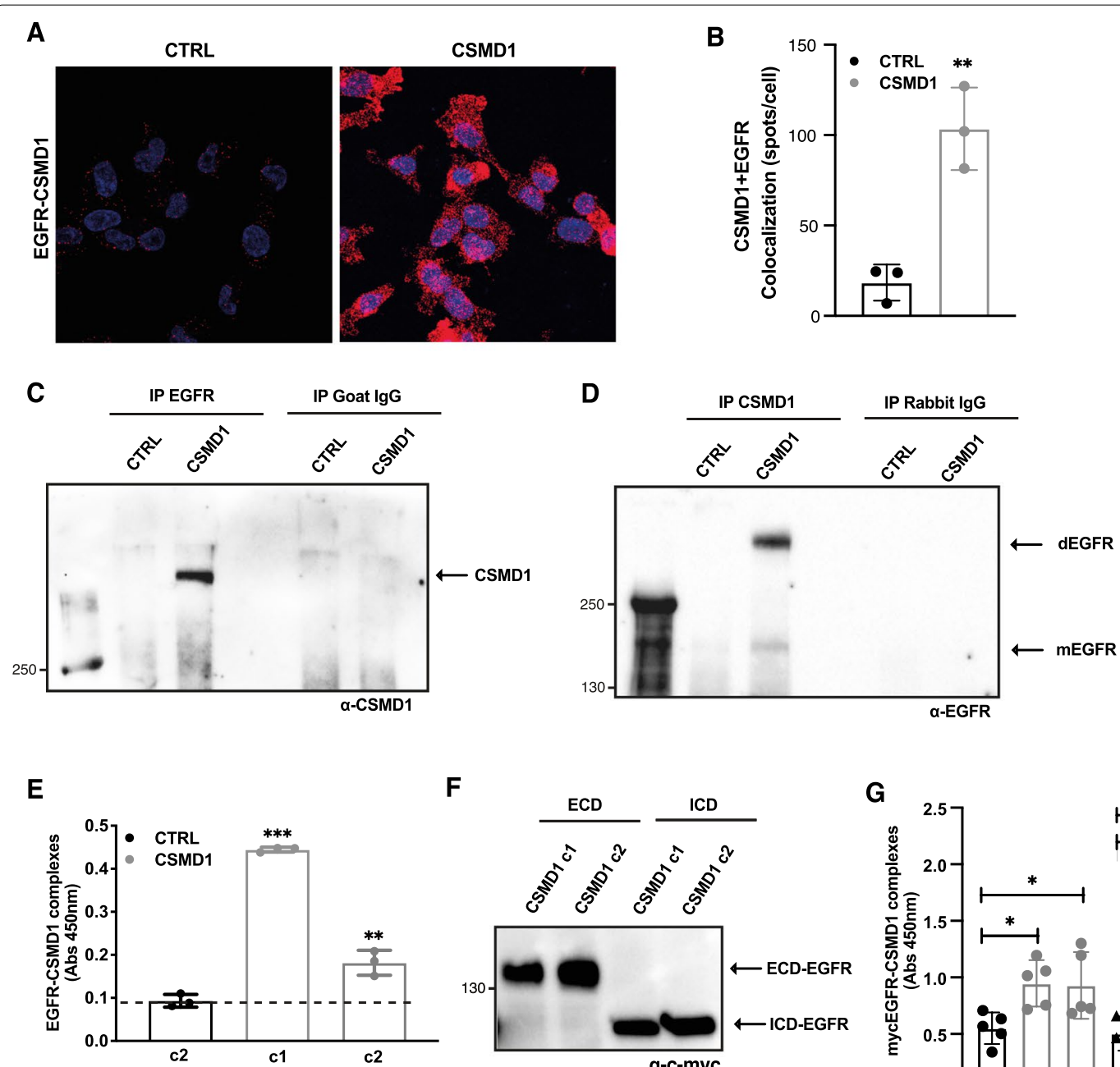

$\mathbf{F}$
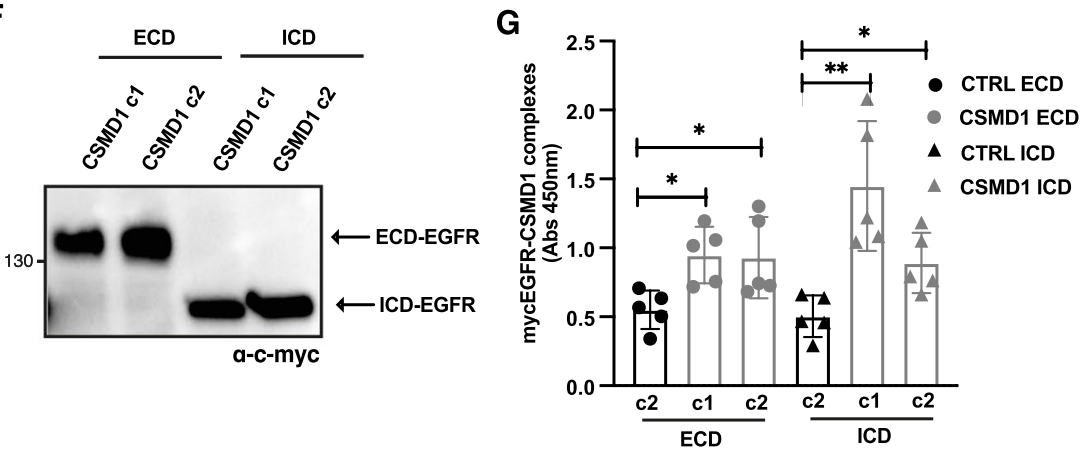

H
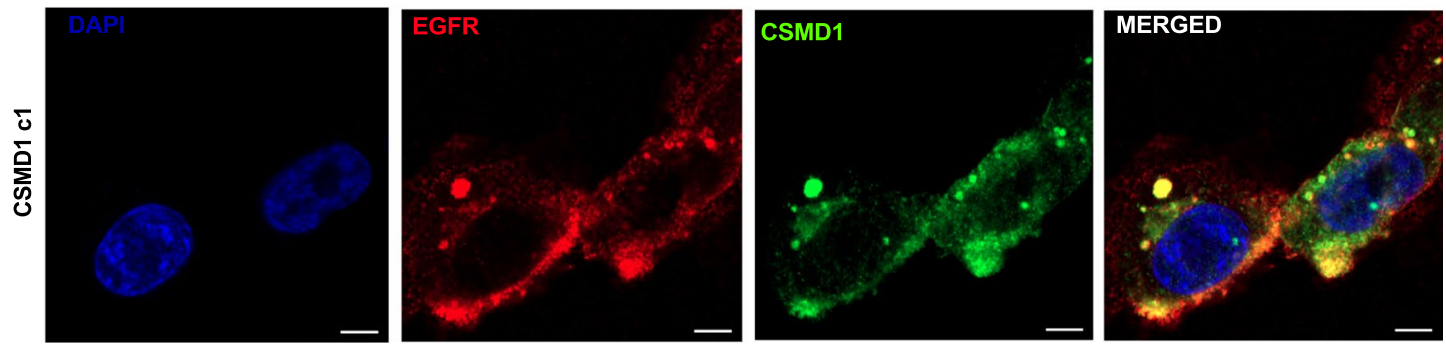

Fig. 2 (See legend on previous page.)

of ECD-EGFR and ICD-EGFR constructs and subsequent expression was confirmed by western blot using an anti-c-myc antibody (Fig. 2F). The interaction of CSMD1 with both ECD-EGFR, as well as ICD-EGFR in two clones differing in CSMD1 expression levels was detected by ELISA, in which complexes were captured with immobilized anti-myc antibodies and detected with anti-CSMD1 antibodies (Fig. 2G). Finally, using confocal microscopy, we documented that CSMD1 co-localizes with EGFR on the cell surface as well as in spotted structures resembling trafficking vehicles (Fig. 2H). 
EGFR signaling is decreased in the presence of CSMD1

To determine EGFR activation in the presence of CSMD1, EGFR was immunoprecipitated in EGF-stimulated CTRL and CSMD1 expressing MDA-MB-231 cells and overall phosphorylation levels were examined by immunoblotting for phosphotyrosine and phosphoserine residues. As expected, EGF induced overall tyrosine and serine phosphorylation of EGFR in the CTRL clones. In contrast, CSMD1-expressing clones exhibited markedly less overall tyrosine and serine phosphorylation in both CSMD1-expressing clones (Supplementary Fig. 1F-I).

Apart from EGF, EGFR can be activated by numerous structurally related ligands with different binding affinities and subsequent signaling kinetics [29, 30]. Thus, we evaluated the effect of CSMD1 expression on EGFR phosphorylation at residue Y1068, known to correlate with EGFR kinase activation, while stimulating with two high affinity ligands EGF $(25 \mathrm{ng} / \mathrm{mL})$ and TGF $\alpha(25 \mathrm{ng} / \mathrm{mL})$, as well as a weaker affinity ligand, AREG (100 ng/mL). In all cases, CSMD1 expression in the MDA-MB-231 cells decreased the activation of EGFR at Y1068 in response to its natural ligands, especially TGF $\alpha$ and AREG (Fig. 3A). EGF-induced EGFR activation increased in a time-dependent manner in the CTRL cells, in contrast with CSMD1-expressing cells in which EGFR activation was delayed and did not reach the same levels as in CTRL cells, after 30 min stimulation (Fig. 3B-D). In the case of CTRL cells, EGFR expression was downregulated during EGF stimulation, whereas in CSMD1-expressing cells the EGFR level was stable and downregulated in a ligand-independent manner (Fig. 3B \& E).

Active EGFR initiates several signaling cascades. Thus, we next assayed the activation status of key downstream effectors arising upon EGF stimulation. Specifically, in CSMD1-expressing cells we detected a diminished AKT phosphorylation at both Ser473 and Thr308 residues at $15 \mathrm{~min}$ and $30 \mathrm{~min}$ EGF stimulation, in comparison with CTRL cells (Fig. 3F-J). This indicates a possible effect of CSMD1 in PI3K through the inhibition of EGFR activation.

Taken together, the EGF-induced EGFR signaling axis is affected by the presence of CSMD1, while it appears to be possibly accompanied by an altered endocytic trafficking fate of the receptor.

\section{CSMD1 governs EGFR endosomal trafficking fate}

Binding of EGF to EGFR leads to internalization of the receptor and trafficking via the endocytic pathway. Binding assays with ${ }^{125}$ I-labeled EGF indicated that CSMD1expressing cells exhibit a similar binding affinity to EGF as CTRL cells (Supplementary Fig. 1J).

Since we observed lower EGFR levels and altered dynamics of EGFR downstream signaling in CSMD1expressing cells, we investigated whether CSMD1 contributes to the receptor turnover and stability. First, we monitored EGFR dimerization upon EGF stimulation in CTRL and CSMD1 MDA-MB-231 cells by cross-linking the cell surface proteins and subsequently detecting the formed EGFR dimers induced by EGF stimulation. EGFR monomers and dimers were detected by western blotting with antibodies detecting EGFR, while treatment with Gefitinib (GEF), a tyrosine kinase inhibitor, was used as a positive control, as it is reported in the literature to induce EGFR dimerization [26]. EGF stimulation induced EGFR dimerization in CTRL cells, whereas CSMD1-expressing cells exhibited a decreased rate of EGFR dimerization, even upon treatment with GEF (Fig. 4A \& B).

EGFR signaling can also be modulated by the posttranslational modification ubiquitination. To address EGFR trafficking in the presence of CSMD1, whole lysates of MDA-MB-231 CTRL and CSMD1 cells in denaturing and minimally denaturing conditions were subjected to immunoprecipitation of EGFR, and ubiquitination was monitored using immunoblotting for ubiquitin (Ub). High levels of EGFR ubiquitination were detected in the presence of CSMD1, both in unstimulated and stimulated conditions (Supplementary Fig. 2A). In

\footnotetext{
(See figure on next page.)

Fig. 3 EGFR signaling cascade in the presence of CSMD1. A Representative western blots of total lysates CTRL and CSMD1 MDA-MB-231 cells immunodetecting phosphorylated EGFR at the residue Y1068 (pEGFR Y1068) and total EGFR with $\beta$-tubulin used as a loading control upon stimulation with EGF (25 ng/mL), TGF-a $(25 \mathrm{ng} / \mathrm{mL})$ and AREG (100 ng/mL) for $30 \mathrm{~min}$. CTRL and CSMD1 MDA-MB-231 cells were serum starved for $2 \mathrm{~h}$ and then treated with $25 \mathrm{ng} / \mathrm{mL}$ EGF for the indicated time points (5, 15 and $30 \mathrm{~min})$. Unstimulated cells were also included in the experiment. B Immunoblot analysis of phosphorylated EGFR at the residue Y1068, total EGFR and $\beta$-tubulin used as a loading control. C Densitometry of pEGFRY1068 normalized to total EGFR. D Densitometry of pEGFRY1068 normalized to $\beta$-tubulin, and (E) densitometry of total EGFR normalized to $\beta$-tubulin. $\mathbf{F}$ Immunoblot analysis of phosphorylated Akt at the residues Ser473 (pAkt Ser473) as well as Thr308 (pAkt Thr308), total Akt and GAPDH used as a loading control. G Densitometry of pAkt Ser473 normalized to total Akt. H Densitometry of pAkt Ser473 normalized to GAPDH, (I) densitometry of pAkt Thr308 normalized to total Akt and (J) densitometry of pAkt Thr308 normalized to GAPDH. All experiments were repeated at least 3 times with bars indicating mean $\pm S D$, black and grey circles correspond to independent data points for CTRL and CSMD1 groups, respectively. A two-way ANOVA Bonferroni's multiple comparisons test was used when comparing 3 or more groups with 2 variables $\left(^{*}<0.05\right.$, $\left.{ }^{* *}<0.01,{ }^{* *}<0.001,{ }^{* * *}<0.0001\right)$
} 
A

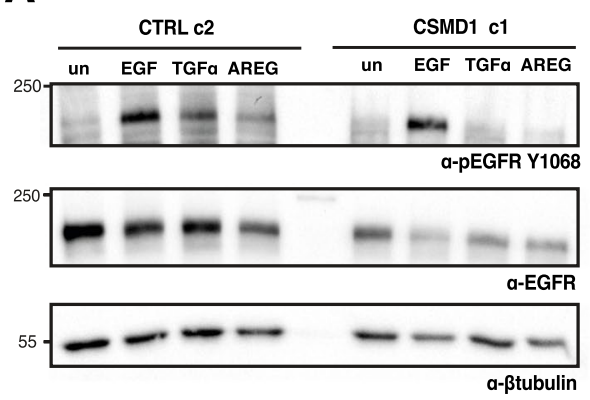

B
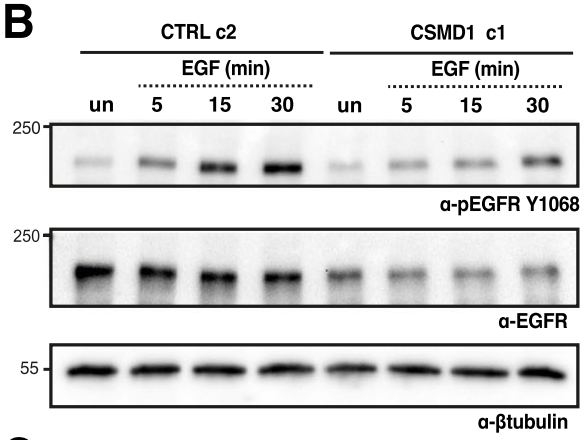

C

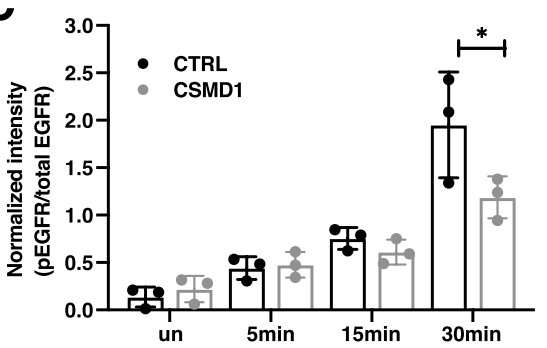

D

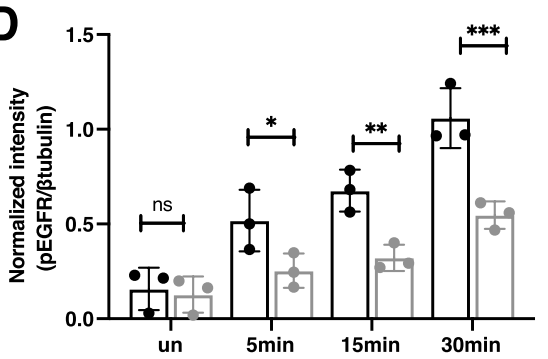

E

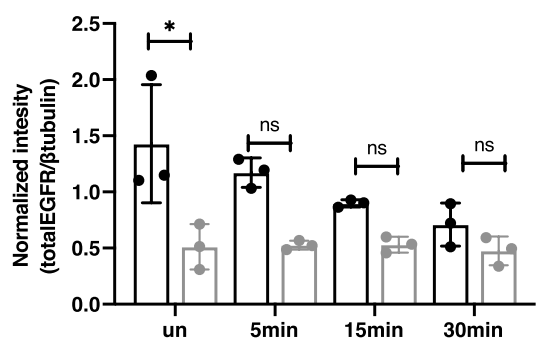

F

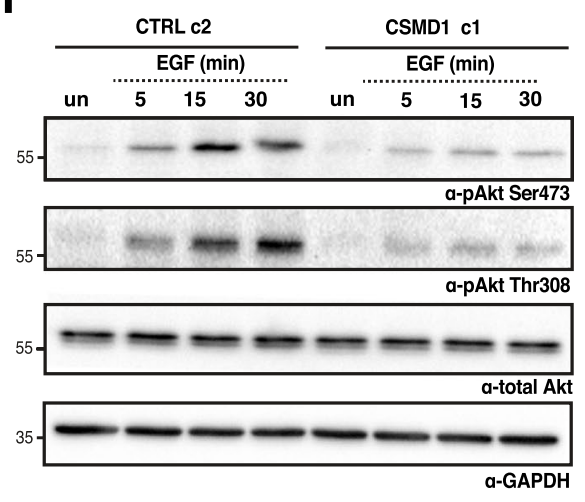

G

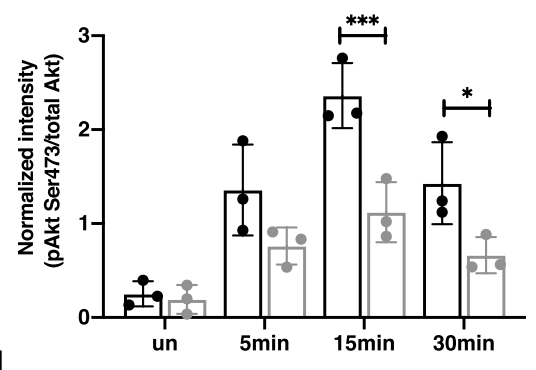

H

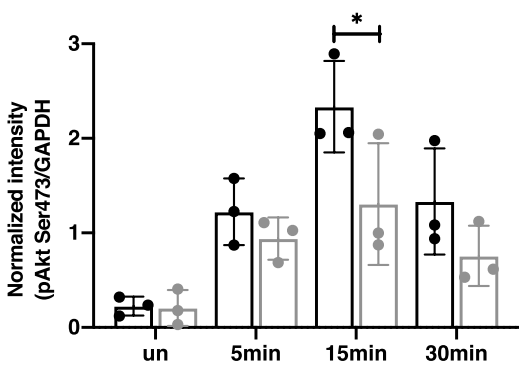

I

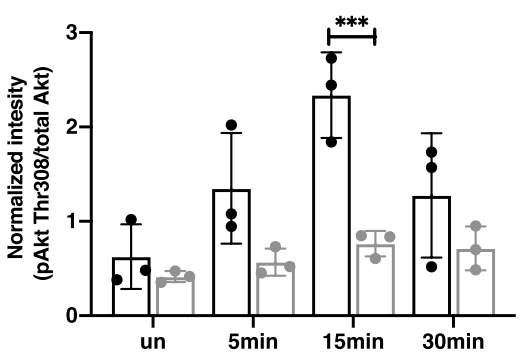

J

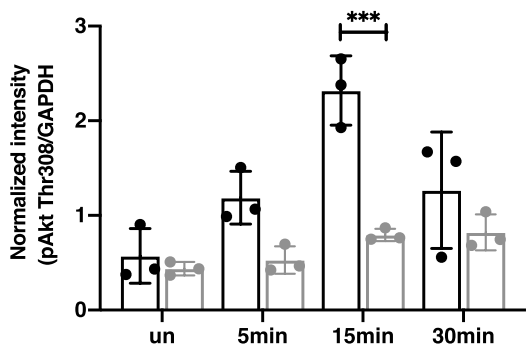

Fig. 3 (See legend on previous page.) 
addition, when analyzing minimally denaturing lysates, we observed a high recruitment of Ub-tagged proteins to EGFR in the presence of CSMD1 (Fig. 4C).

The two fates of EGFR after the binding of EGF are either degradation or recycling [31]. Therefore, we firstly investigated if CSMD1 affects the EGF-induced EGFR degradation by treating MDA-MB-231 CTRL and CSMD1 overexpressing cells with EGF for 0, 4, 8, $12 \mathrm{~h}$ followed by measurements of total EGFR levels in the obtained lysates. To eliminate the impact of de-novo EGFR synthesis, both CTRL and CSMD1 cells were pretreated with the translational inhibitor cycloheximide (CHX) before and during the EGF stimulation. CSMD1 increased the EGFR degradation rate, as in CSMD1expressing cells the EGFR half-life $(8 \mathrm{~h})$ was shorter than in CTRL cells (12 h; Fig. 4D \&E). On the other hand, the internalization rate of ${ }^{125}$ I-labeled EGF was comparable between CTRL and CSMD1-expressing cells (Supplementary Fig. 2B). Therefore, we hypothesized that CSMD1 may direct EGFR towards degradation by ubiquitination, thus modulating its trafficking.

Proteasomal and lysosomal degradation systems are the main drivers of EGFR degradation. To evaluate which system is responsible for the increased EGFR degradation in CSMD1-expressing cells, we performed similar degradation experiments as described above in the presence of a lysosomal inhibitor $\left(\mathrm{NH}_{4} \mathrm{Cl}\right)$, or proteasomal inhibitor (MG132). Our results revealed that increased EGFR degradation in the presence of CSMD1 was reverted only when lysosomal activity was inhibited (Fig. 4F), while proteasome inhibition did not alter EGFR degradation levels (Fig. 4G). EGFR recovery in the presence of inhibitors was also calculated, highlighting the higher EGFR recovery upon treatment with $\mathrm{NH}_{4} \mathrm{Cl}$ in the CSMD1expressing cells compared to CTRL, whereas no effect of MG132 on EGFR recovery was observed (Fig. 4H). To further support this observation, we investigated EGFR localization by confocal microscopy in CTRL and
CSMD1 MDA-MB-231 cells, using early and late endosomal markers, Early Endosome Antigen 1 (EEA1) (Fig. 4I) and Lysosomal-associated membrane protein 1 (LAMP1) (Fig. 4M), respectively. Following $15 \mathrm{~min}$ and $120 \mathrm{~min}$ EGF stimulation, colocalization between EGFR and/or EEA1 and LAMP1 was barely observed in CTRL cells, while it was increased in CSMD1-expressing cells, especially in the case of late endosomal marker, suggesting that CSMD1 plays a fundamental role in EGFR trafficking (Fig. 4J \& N). In addition, cell fractionation into cytosol and membrane extracts revealed a shift of EGFR from the membrane to the cytosolic fractions in CSMD1-expressing cells compared to CTRL cells, in both unstimulated and EGF-stimulated conditions (Supplementary Fig. 2C \& D), supporting increased internalization/degradation in CSMD1-expressing cells. On the other hand, CSMD1 itself highly colocalized with both EEA1 and LAMP1, but only in the case of LAMP1 was the colocalization significantly increased upon 120 min EGF stimulation (Fig. 4K \& O). Interestingly, EGFR-CSMD1 colocalization was also significantly increased after 120 min EGF stimulation (Fig. 4L). In addition, a stronger triple colocalization of EGFR-CSMD1-EEA1 and weaker EGFR-CSMD1LAMP1 colozalisation were detected in CSMD1-expressing cells.

Key findings reported in this study were confirmed in two additional triple negative breast cancer cell lines, BT-20 and MDA-MB-468. Firstly, we documented CSMD1-EGFR interaction in BT-20 cells by co-immunoprecipitation (Supplementary Fig. 3A). However, in contrast with MDA-MB-231 cells, in immunoprecipitated CSMD1-complexes we detected a stronger signal approximately corresponding to the size of EGFR monomer and a weaker signal of EGFR dimers, while no such signals were detected with the corresponding isotype control antibodies. In BT-20 and MDA-MB-468 cells, even though EGF-induced EGFR phosphorylation was not markedly affected (Supplementary Fig. 3B-E \&

\footnotetext{
(See figure on next page.)

Fig. 4 EGFR endocytic trafficking in the presence of CSMD1. A Detection of EGFR dimers upon EGF ( $25 \mathrm{ng} / \mathrm{ml}$ for $15 \mathrm{~min})$ and GEF (10 $\mu \mathrm{M})$ stimulation followed by crosslinking with $\mathrm{BS}^{3}$. B Densitometry of dimers of EGFR normalized to ßtubulin (C) CSMD1 triggers EGFR ubiquitination which was detected using EGFR immunoprecipitation followed by immunoblotting with anti-Ub antibody. Representative blots from three independent experiments are shown. D Representative immunoblots of EGFR levels in lysates of MDA-MB-231 CTRL and CSMD1 overexpressing clonal cells, pre-treated with translation inhibitor cycloheximide (CHX) $(100 \mu \mathrm{g} / \mathrm{mL})$ for $2 \mathrm{~h}$ and treated with EGF $(25 \mathrm{ng} / \mathrm{ml})$ for $0,4,8,12 \mathrm{~h}$ (h). E Quantification of EGFR levels in MDA-MB-231 cells plotted against time. Representative immunoblots of EGFR levels in CTRL and CSMD1 overexpressing cells, pre-treated with $\mathrm{CHX}(100 \mu \mathrm{g} / \mathrm{mL})$ for $2 \mathrm{~h}$ followed by treatment with $25 \mathrm{ng} / \mathrm{mL}$ EGF in the presence of $(\mathbf{F}) \mathrm{NH}_{4} \mathrm{Cl}$ and $(\mathbf{G})$ MG132 for $12 \mathrm{~h}$. $\mathbf{H}$ Quantification of EGFR recovery in both $\mathrm{NH}_{4} \mathrm{Cl}$ or MG132 treated CSMD1 and CTRL cells. I Representative confocal images of CTRL and CSMD1 BCCs co-stained for EGFR (red), CSMD1 (blue), EEA1 (green), nucleus (SYTOX orange). Quantification of colocalization of (J) EGFR-EEA1, (K) EEA1-CSMD1 and (L) CSMD1-EGFR complexes. M Representative confocal images of CTRL and CSMD1 BCCs co-stained for EGFR (red), CSMD1 (blue), LAMP1 (green), nucleus (SYTOX orange). Quantification of colocalization of (N) EGFR-LAMP1 and (O) LAMP1-CSMD1 complexes. Scale bars $5 \mu \mathrm{m}$. All experiments were repeated at least 3 times with bars indicating mean \pm SD, grey circles correspond to independent data points for CTRL and CSMD1 groups, respectively. A two-way ANOVA Bonferroni's multiple comparisons test was used when comparing CTRL and CSMD1 groups $\left({ }^{*}<0.05,{ }^{* * * *}<0.0001\right)$. A one-way ANOVA Dunnetts's multiple comparisons test was used when comparing CSMD1 cells in different time-points $\left({ }^{*}<0.05,{ }^{* * *}<0.0001\right)$
} 

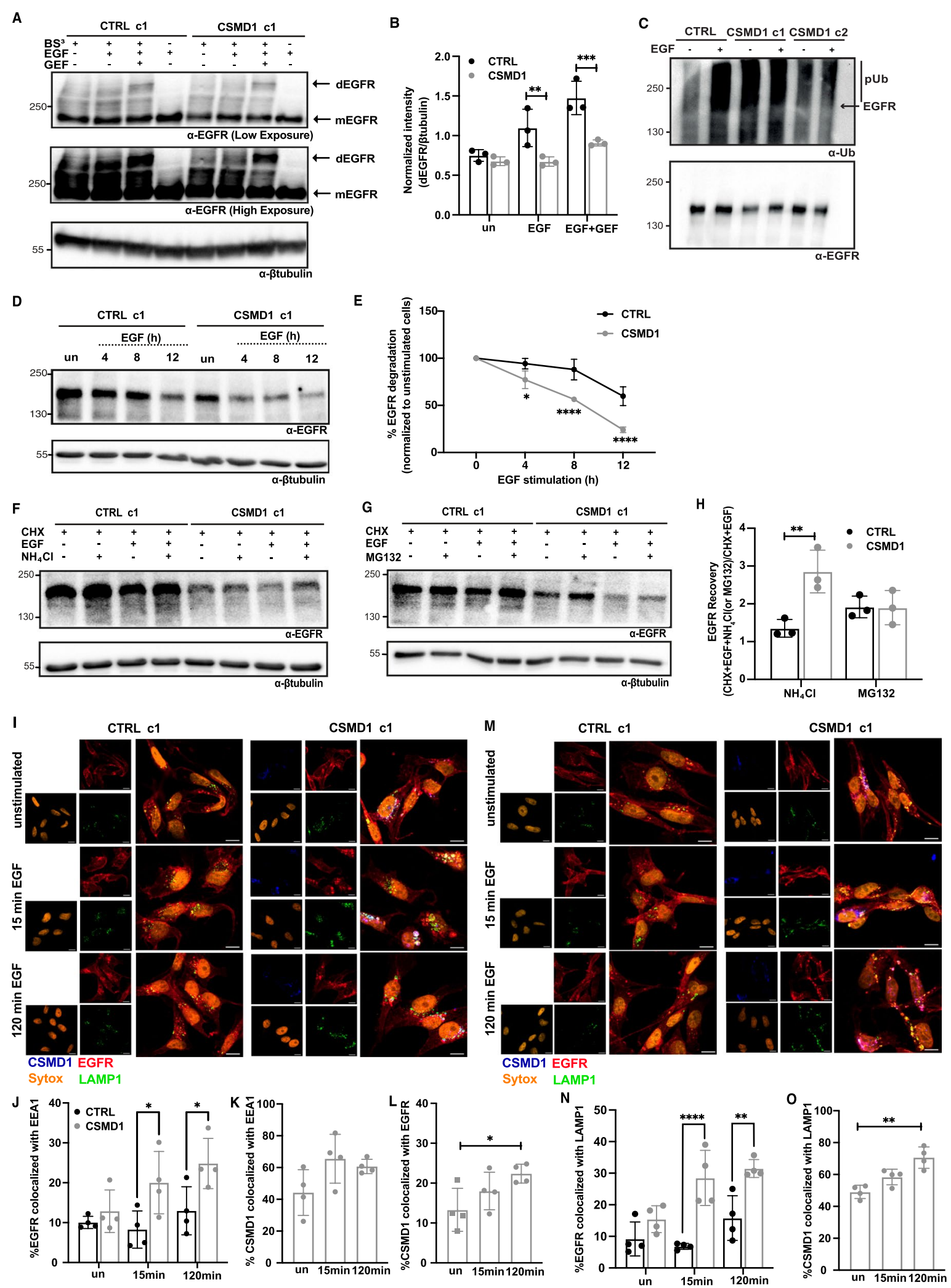

Fig. 4 (See legend on previous page.) 
Supplementary Fig. 4A-D), the PI3K-AKT cascade was also inhibited by CSMD1 upon EGF stimulation, similar to MDA-MB-231 BBCs. Specifically, activation of AKT at the Ser473 residue was less pronounced in CSMD1expressing BT-20 and MDA-MB-468 cells than CTRL cells, at shorter time points (Supplementary Fig. 3F-H \& Supplementary Fig. 4E-G). In addition, EGF-induced EGFR degradation in BT-20 and MDA-MB-468 CSMD1 cells was faster in comparison to CTRL cells, suggesting that CSMD1 acts on EGFR action via multiple mechanisms (Supplementary Fig. 3I \& J \& Supplementary Fig. 4H-I).

\section{CSMD1 expression increases sensitivity to chemotherapy}

We hypothesized that CSMD1-bearing tumors are sensitive to common breast cancer chemotherapy agents and that CSMD1 expression could therefore be used as a predictive marker of response to therapy. To address this we treated MDA-MB-231 CTRL and CSMD1 cells with increasing doses of chemotherapy agents and monitored drug-induced apoptosis by annexin $\mathrm{V}$ staining, and live/dead cell discrimination with Zombie aqua staining, using flow cytometry (Fig. 5A \& B). The chemotherapy agents tested, doxorubicin (a topoisomerase activity inhibitor), epirubicin (an anthracycline drug), docetaxel (a microtubule inhibitor), as well as 5-fluorouracil (pyrimidine analog antimetabolite) were not effective in killing the TNBC MDA-MB-231 CTRL cells in the range of concentrations used, as documented by the percentage of late apoptotic cells. In contrast, CSMD1 MDA-MB-231 expressing cells were sensitive to doxorubicin-, epirubicin- and 5-fluorouracil-induced apoptosis in a dose dependent manner, while docetaxel was less effective, showing a trend in the lower doses and a significant killing effect in the highest dose used (Fig. 5B). Additionally, caspase-3 activity, monitored by the levels of cleaved caspase-3, was significantly upregulated in the CSMD1 cells treated with doxorubicin and epirubicin in comparison with CTRL cells, corresponding well with the increased observed numbers of apoptotic cells (Fig. 5C \& D). In line with this observation, BT-20 and MDA-MB-468 CSMD1-overexpressing cells also exhibited increased sensitivity to the chemotherapy drugs doxorubicin and epirubicin (Supplementary Fig. 3 K \& Supplementary Fig. 4J).

In breast cancer, several mechanisms of drug resistance are known, including autophagy and drug efflux/ uptake. In our experimental setup, no statistical difference was observed between CTRL and CSMD1 BCCs in autophagy induction upon chemotherapy treatment, as monitored by conversion of LC3BI to LC3BII (Fig. 5C). However, a doxorubicin efflux assay indicated that drug efflux activity or uptake were impaired in the presence of CSMD1, resulting in increased intracellular drug availability (Supplementary Fig. 5A\&B). Even though EGFR overexpression is a common feature of TNBC, tyrosine kinase inhibition monotherapy is not effective. Pretreatment of the BCCs with the tyrosine kinase inhibitor GEF followed by low doses of doxorubicin or epirubicin $(1 \mu \mathrm{M})$ did not overcome resistance of CTRL cells to chemotherapy, while CSMD1-expressing cells exhibited a significant increase in the percentage of late apoptotic cells, comparable to the highest concentration of chemotherapy agent used in the monotherapy experimental setup (Fig. 5E). This effect was not reversed when blocking autophagy with chloroquine, and pretreatment with GEF did not affect autophagy levels (Supplementary Fig. 5C). Taken together, CSMD1 inhibits chemoresistance of BBCs by affecting intracellular drug availability, rather than autophagy.

To strengthen our findings and provide a better representation of the in vivo environment [32], we generated uniform tumorspheres of MDA-MB-231 and BT-20 cells. Similar to the two dimensional cell culture, CSMD1-expressing cells were more sensitive to chemotherapy treatment, especially in the case of MDA-MB-231 (Fig. 6A), whereas BT20 cells were more resistant in general (Fig. 6B). Further, pretreatment of the CSMD1-expressing tumorspheres with the tyrosine kinase inhibitor GEF followed by low doses of doxorubicin or epirubicin overcame the resistance of CTRL cells to chemotherapy, especially in the case of BT-20 cells where only the GEF pretreated CSMD1-expressing cells exhibited a significant increase in the percentage of late apoptotic cells (Fig. 6B). Similar observations were documented for MDA-MB-231 CSMD1 expressing cells,

\footnotetext{
(See figure on next page.)

Fig. 5 CSMD1-expressing BCCs are sensitive to chemotherapy. MDA-MB-231 CTRL and CSMD1 cells were treated with different chemotherapy agents for $48 \mathrm{~h}$, and apoptosis was monitored using annexin $\mathrm{V}$ staining while live/dead cell discrimination was performed with Zombie Aqua staining, both using flow cytometry. Bar graphs showing percentages of (A) early and (B) late apoptotic cells. C Immunoblot analysis of cleaved caspase-3 and LC3B upon treatment with different chemotherapy agents; $\beta$-tubulin was used as a loading control. D Densitometry analysis of cleaved caspase-3 normalized to $\beta$-tubulin. E MDA-MB-231 CTRL and CSMD1 cells were pre-treated with GEF, QC alone and combination of both for $24 \mathrm{~h}$, following doxorubicin and epirubicin $(1 \mu \mathrm{M})$ treatment for $48 \mathrm{~h}$. Bar graphs showing percentages of early and late apoptotic cells. All experiments were repeated at least 3 times with bars indicating mean $\pm S D$, grey circles correspond to independent data points for CTRL and CSMD1 groups, respectively. A two-way ANOVA Bonferroni's multiple comparisons test was used when comparing CTRL and CSMD1 groups in different concentration of chemotherapy drugs or treatments
} 


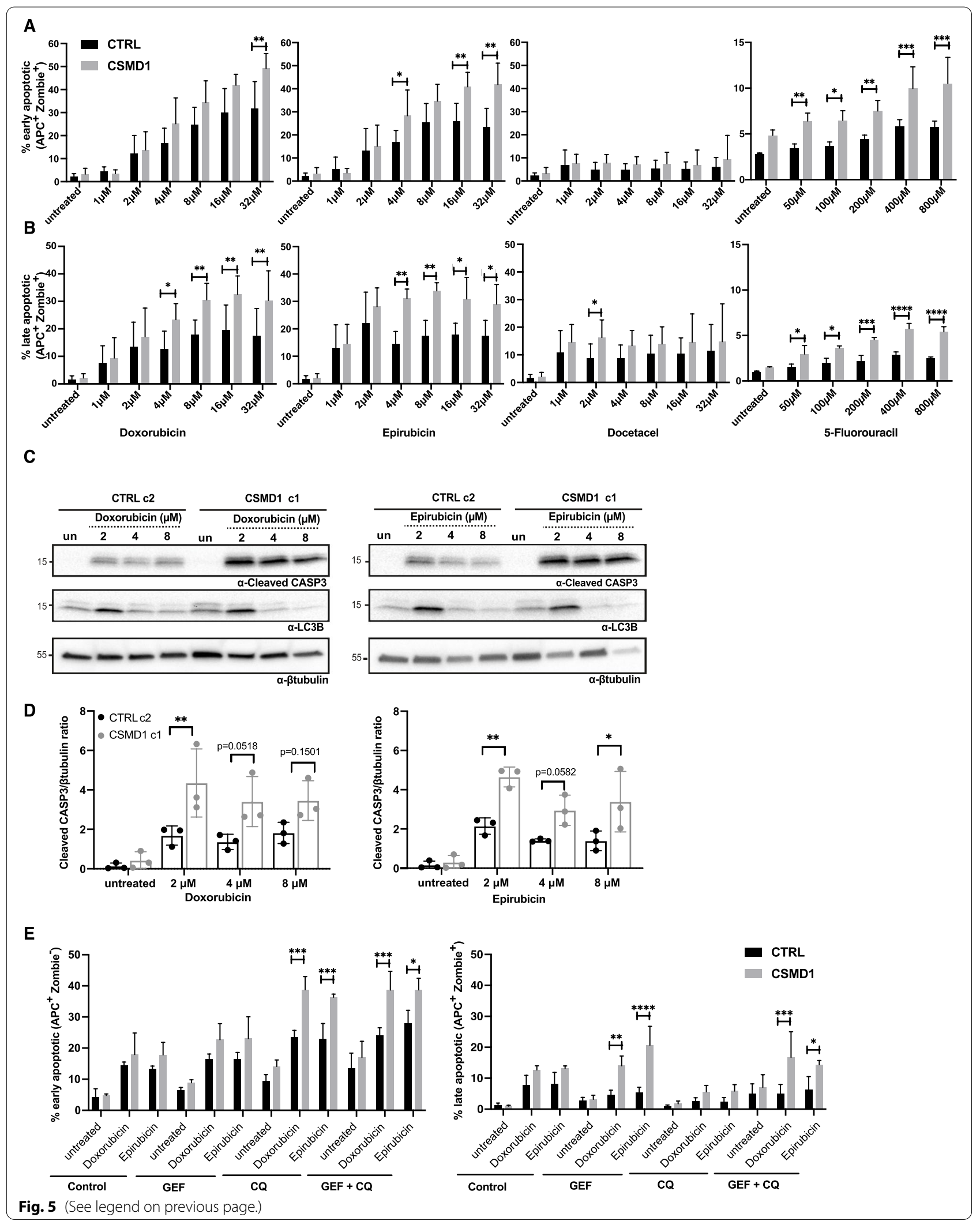



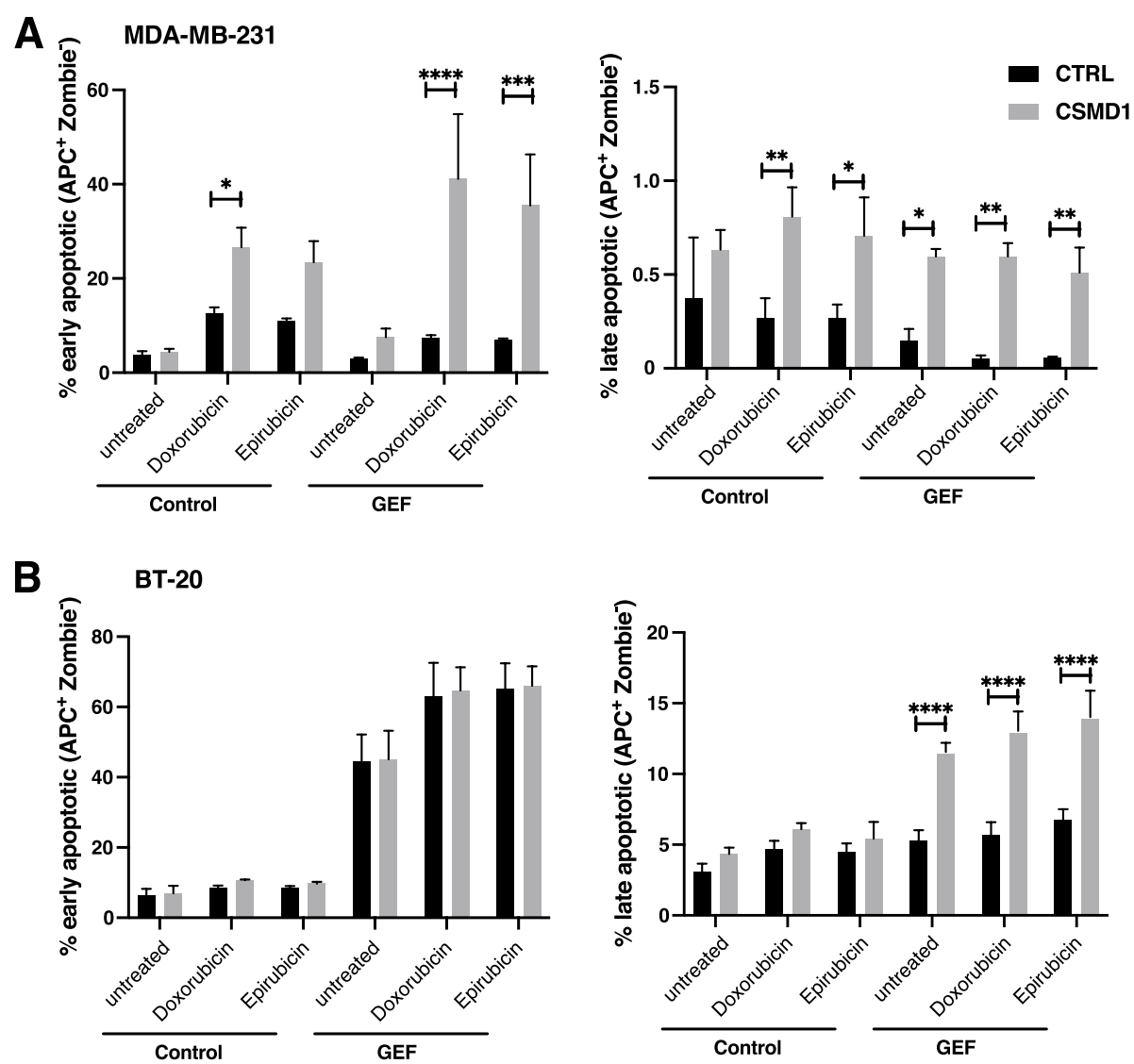

Fig. 6 CSMD1-expressing tumorspheres are more sensitive to chemotherapy agents. A MDA-MB-231 and (B) BT-20 tumorspheres were pre-treated with $35 \mu \mathrm{M}$ of GEF followed by $2 \mu \mathrm{M}$ of doxorubicin or epirubicin for two days (MDA-MB-231) or $8 \mu \mathrm{M}$ of doxorubicin and epirubicin for one day (BT-20). Early and late apoptotic cells were detected using Annexin V and Zombie Aqua staining. Bar graphs showing percentages of early and late apoptotic cells. All experiments were repeated at least 3 times with bars indicating mean \pm SD, grey circles correspond to independent data points for CTRL and CSMD1 groups, respectively. A two-way ANOVA Bonferroni's multiple comparisons test was used when comparing CTRL and CSMD1 groups in different treatments $\left({ }^{*}<0.05,{ }^{* *}<0.01,{ }^{* * *}<0.001\right)$

presenting a markedly higher percentage of early apoptotic cells in the GEF pretreated cells in contrast with CTRL cells (Fig. 6A). In conclusion, CSMD1 protein expression rendered tumorspheres pretreated with EGFR inhibitor more sensitive to chemotherapy.

\section{CSMD1 gene expression levels and association with outcome in breast cancer patients}

In our previous study, we showed that CSMD1 mammary gene expression is dramatically decreased in breast cancer patients compared to normal mammary tissue [28]. In the current study, we expanded our mRNA analyses to a modern population-based cohort, the Swedish Cancerome Analysis Network - Breast (SCAN-B), consisting of 3520 RNA sequenced primary breast cancer patients as reported by [27]. In accordance with previous observations, CSMD1 gene expression (FPKM) was generally low across SCAN-B patients with no bimodal patterns. Of note, this low, unimodal expression makes cut-offs for gene expression defined groups arbitrary (Supplementary Fig. 6A \& B). Moreover, the CSMD1 gene expression profile across clinical subgroups (ER, HER2, and LN status, as well as histological grade) and molecular PAM50 subtypes (Basal, HER2, Luminal A, Luminal B and normallike) of SCAN-B patients was investigated. In all cases, low transcriptional variation was observed across the different subgroups, while there was a range of outliers within each subgroup. Still, CSMD1 gene expression levels showed significant differences between certain clinical subgroups, with e.g. lower levels in HER2-amplified disease and TNBC compared to luminal disease, lower levels in grade 3 tumors compared to grade 1 and 2 tumors, and lower levels in the basal-like, HER2-enriched, and luminal B PAM50 subtypes compared to luminal A tumors (Kruskal-Wallis test $\mathrm{p}<0.00001$, Fig. 7A-B). CSMD1 expression differences were also observed within clinical subgroups, e.g., in TNBC lower mRNA expression was observed in PAM50 basal-like TNBC cases compared to 
non-basal-like TNBC (Mann-Whitney test $\mathrm{p}<0.00001$, Fig. 7C). Difference in CSMD1 expression between basal and non-basal in TNBC was mirrored by a similar trend for EGFR (Mann-Whitney test $\mathrm{p}=0.008$, Supplementary Fig. 6C).

To test the association of CSMD1 gene expression with patient outcome after adjuvant standard-of-care chemotherapy in TNBC, we stratified the 239 adjuvant-treated TNBC patients according to the median expression of CSMD1 and analyzed the two groups using overall survival (OS) and invasive disease-free survival (IDFS) as clinical endpoints. For both endpoints, higher gene expression of CSMD1 tended to be associated with better prognosis, although both log-rank p-values and univariable Cox regression analysis did not reach statistical significance (Fig. 7E \& F).

To assess the independent prognostic value of CSMD1 gene expression in adjuvant treated TNBC patients, we performed multivariable Cox regression analysis adjusting for tumor size $(\leq 20 \mathrm{~mm},>20 \mathrm{~mm})$, tumor grade (2, 3 ), and lymph node status (N0, N+) using OS and IDFS as the clinical endpoints. CSMD1 gene expression above the median did not reach statistical significance $(\mathrm{p}<0.05)$, although similar trends, as in the Kaplan-Meier and univariable Cox analyses, of improved $\mathrm{OS}(\mathrm{HR}=0.63$, 95\% confidence interval $(\mathrm{CI}): 0.32-1.3, \mathrm{p}=0.201)$ and IDFS (HR $=0.63$, 95\% confidence interval (CI): 0.33-1.1, $\mathrm{p}=0.123$ ) were observed.

To test the association of CSMD1 and EGFR gene expression with outcome after adjuvant standard-ofcare chemotherapy in TNBC, we further stratified the 239 adjuvant-treated TNBC patients according to the median expression of CSMD1 and EGFR, and analyzed the four groups using overall survival (OS) and invasive disease-free survival (IDFS) as clinical endpoints. For both endpoints, higher gene expression of CSMD1 together with low gene expression of EGFR tended to be associated with better prognosis, although both log-rank p-values and univariable Cox regression analysis did not reach statistical significance. In contrast, lower gene expression of CSMD1 together with high gene expression of EGFR tended to be associated with worse prognosis (Fig. 7G \& H).

Collectively, in accordance with our in vitro data, a trend of higher chemosensitivity in CSMD1-expressing tumors appears to be evident in a population-based cohort of TNBC. Moreover, CSMD1 gene expression differed between basal-like and non-basal-like TNBC, suggesting a connection to underlying biological differences between these subgroups. Noteworthy, EGFR expression together with CSMD1 further characterizes subsets of $\mathrm{BC}$ patients with varying chemosensitivity.

\section{Discussion}

TNBC is an aggressive breast cancer subtype, characterized by the lack of expression of estrogen receptor (ER), progesterone receptor, and human epidermal growth factor receptor 2 (HER2). There are no clinically approved targeted therapies for TNBC, and thus, there is an urgent need to identify potent, highly effective therapeutic targets. EGFR is of immediate medical and biological importance due to its well-established roles in developmental biology, tissue homeostasis, and cancer [33]. Overexpression of EGFR has been reported in $15-20 \%$ of all breast carcinomas and in $50-70 \%$ of TNBCs [34, 35]. It is known that breast cancers with high EGFR expression are more aggressive, larger in size and more capable to metastasize to the lymph nodes and brain. Additionally, patients with EGFR-positive tumors have a worse overall, disease free and post-relapse survival after hormonal and/or chemotherapy, lacking prognostic indicators [36, 37]. However, EGFR-targeted therapy has poor performance in TNBC [37]. In the current study, we report for the first time the direct interaction of EGFR with the tumor suppressor CSMD1, which leads to attenuation of EGFR signaling due to altered trafficking of the receptor. Importantly, CSMD1-expressing cells are as a result more sensitive to chemotherapy.

To date, we and others have presented evidence that CSMD1 acts as a tumor suppressor in breast cancer, but molecular mechanisms underlying this function of the protein have not been elucidated [28, 38]. Since CSMD1 is a poorly studied protein, we resorted to general screens such as proteome oncology arrays to obtain insights into its cellular effects. Scanning electron microscopy revealed large phenotypic changes in CSMD1-expressing cells that translated into a distinct proteomic signature, rendering them less aggressive. Specifically, proteome profiling showed significant changes in expression of CapG and EpCAM that are both involved in organization

\footnotetext{
(See figure on next page.)

Fig. 7 Gene-set tumor analysis of CSMD1 expression using 3520 breast cancer patients SCAN-B cohort. Box plot of CSMD1 gene expression for tumor samples of SCAN-B cohort stratified according to (A) clinical groups, (B) tumor grade (Nottingham Histological Grade) (C) PAM50 subtypes and (D) box plot of CSMD1 gene expression for tumor samples of SCAN-B cohort subset of TNBC stratified according to basal and non-basal like properties. Kaplan-Meier analysis, using (E) OS and (F) IDFS as endpoints, for TNBC patients treated with chemotherapy $(n=239)$ stratified into the two quantiles based on CSMD1 median gene expression level. Kaplan-Meier analysis, using (G) OS and (H) IDFS as endpoints, for TNBC patients treated with chemotherapy $(n=239)$ stratified into the four quantiles based on CSMD1 and EGFR median gene expression level
} 


\section{A SCAN-B Clinical Groups}

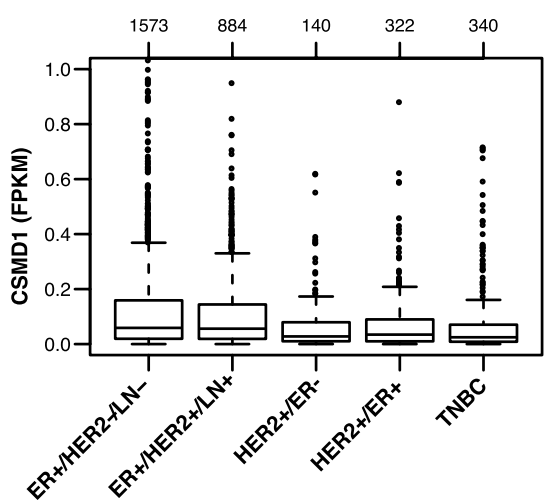

\section{Scan-b Pam50}

(Kruskal-Wallis $\mathrm{p}=\mathbf{1 . 9 e - 1 0 6 )}$

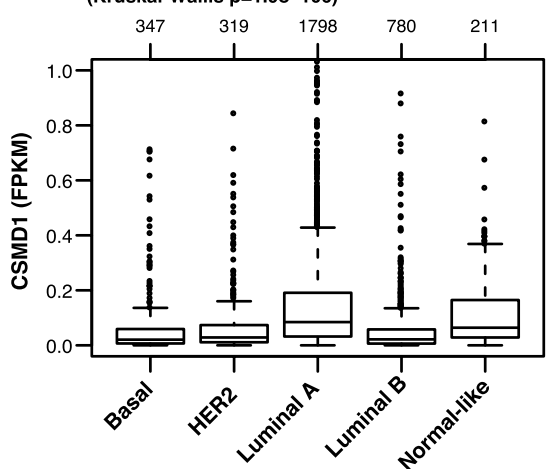

E

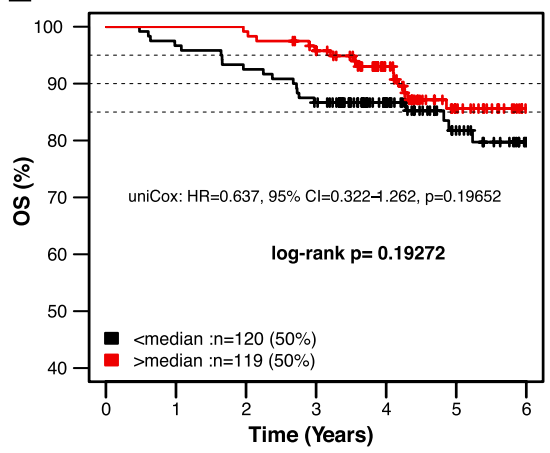

G

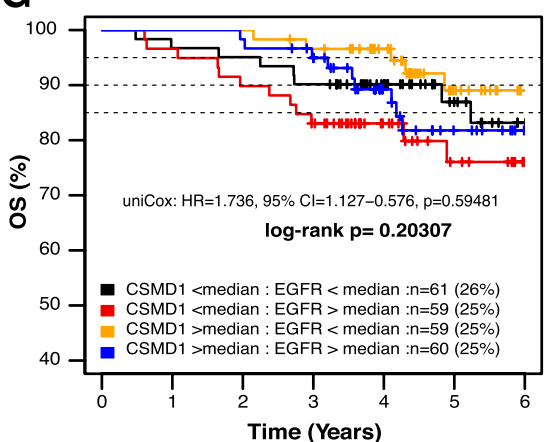

B SCAN-B Histological Grade

(Kruskal-Wallis $p=1.9 e-68$ )

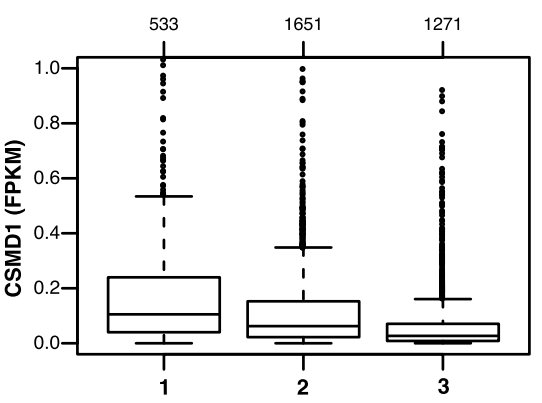

D SCAN-B TNBC Basal vs non-basal (Mann-Whitney $\mathrm{p}=4.82 \mathrm{e}-07$ )

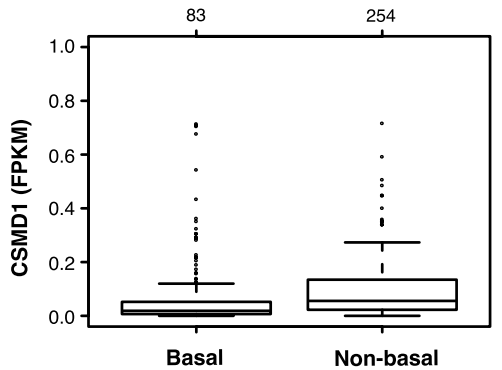

$\mathbf{F}$

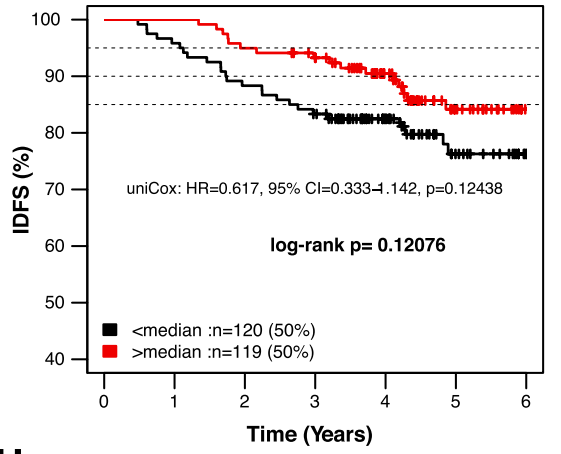

H

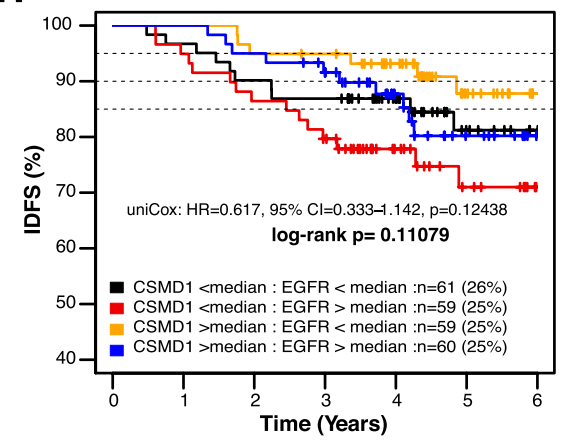

Fig. 7 (See legend on previous page.) 
of the actin cytoskeleton, which could be related to an observed effect on cell motility [39, 40]. In addition, CTSS protein expression was decreased by CSMD1, reflecting the low metastatic potential of these cells. Recently, CTSS was introduced as a potential biomarker in TNBC, while mechanistically contributing to BCC's ability to metastasize to the brain via a Src dependent mechanism [41, 42]. In this study, we focused on the relation between CSMD1 and EGFR, considering the importance of EGFR in TNBC. The motivation behind this was that in the presence of CSMD1, we documented that EGFR overall expression, as well as cell surface expression, was markedly altered, even though its gene expression/mRNA level was not affected.

Upon binding of the ligand, EGFR, which is otherwise in a monomeric, inactive state, aggregates at the cell surface forming homodimeric or heterodimeric complexes, usually with other members of HER family or other receptors. Collectively in this study, several methods were used to show direct interaction between CSMD1 and EGFR, which appears to be mediated by both the extracellular and intracellular portions of EGFR. That interaction leads to diminished formation of EGFR dimers on the cell surface, while EGFR is strongly ubiquitinylated. The tyrosine kinase inhibitor GEF has been reported to induce dimer formation by enhancing the affinity of EGF-EGFR, while lapatinib stabilizes the inactive form of EGFR and reduces the affinity of EGF-EGFR interaction, having no effect in dimer formation [26]. Ubiquitin-tagged EGFR is internalized and trafficked through the endocytic pathway, accompanied by other ubiquitin-tagged intracellular effectors, ultimately targeting the receptor for lysosomal degradation, thereby ensuring termination of the signal [43]. Bearing these facts in mind, CSMD1 seems to interfere with the formation of EGFR dimers and directs UbEGFR in general to a rapid endosomal trafficking. Of note, this rapid endosomal trafficking does not seem to be driven by EGF-EGFR affinity and internalization rate, as both these aspects were similar in CSMD1-expressing and CTRL cells. The CSMD1-EGFR interaction in turn, results in a decreased EGFR intracellular kinase domain phosphorylation, and subsequent interference with the PI3K-AKT signaling cascade downstream of EGFR. A recent study in head and neck squamous cell carcinomas highlighted that genomic aberrations including EGFR amplifications, AKT1 amplifications and CSMD1 deletions, but not PIK3CA, were highly associated with responsiveness to PI3K-targeted drugs [44]. In our experimental setup in three different TNBC cell lines, EGF-EGFR induced AKT activation was abrogated in the presence of CSMD1, even in BT-20 BCCs that harbors an activating mutation in PIK3CA [45]. However, in several clinical trials, PIK3CA mutations are considered as a treatment predictive biomarker and mutation status is an inclusion criterion [46].

Upon EGFR activation, cell surface active receptors are internalized to the early endosomes, where they are sorted either for degradation to terminate the signal, or recycled back to the cell surface to sustain the signal [47]. Intracellular protein degradation occurs most commonly through two major mechanisms, lysosomal degradation and proteasome mediated degradation. Lysosomal degradation occurs through proteolytic enzyme activity within lysosomes while proteasomal degradation is targeted through the ubiquitination of the specific protein. In the case of EGFR, convincing evidence has been reported for ubiquitin-dependent targeting of EGFR to lysosomal degradation. Even though EGFR as such is not a proteasomal target, it has been reported that ubiquitination and proteasomal activity are required for its lysosomal sorting [48]. Our results revealed that CSMD1 directed EGFR preferably to the lysosomal degradation pathway, rather than the proteasomal system. In the presence of CSMD1, EGFR localized faster to the endosomal compartments, while CSMD1 was more strongly associated with the late endosomal marker LAMP1, consistent with the faster degradation rate of EGFR in CSMD1-expressing BCCs.

The main treatments for breast cancer include surgery, chemotherapy, radiotherapy, hormonal therapy as well as targeted cancer drugs. Chemotherapy is introduced to breast cancer treatment as neoadjuvant chemotherapy aiming to shrink locally advanced tumors before surgery, as adjuvant chemotherapy after surgical intervention to eliminate residual cancer cells, as well as a treatment option for advanced metastatic breast cancers [49]. Moreover, inhibition of EGFR signaling is associated with decreased drug efflux activity [50]. In the case of TNBC, which does not respond to hormonal and targeted therapy, the main line of treatment is chemotherapy. In addition, a recent large-scale pharmacogenomics study reported co-occurring resistance between EGFR-RTK inhibitors and chemotherapy in breast cancer [51]. Therefore, it is of significant interest that expression of CSMD1 renders TNBC BCCs more sensitive to chemotherapy in both 2D and 3D cell cultures. In addition, CSMD1 expression increases the effectiveness of combination therapies with first line tyrosine kinase inhibitors and second line treatment chemotherapy at low doses, versus monotherapy treatment. The current study is limited by the lack of in vivo xenografts supporting the interplay between CSMD1 and EGFR axis. These data, albeit suggested by the reviewers, could not be generated due to strict restrictions imposed by Lund University on animal experimentation during Covid pandemic at the period of the 


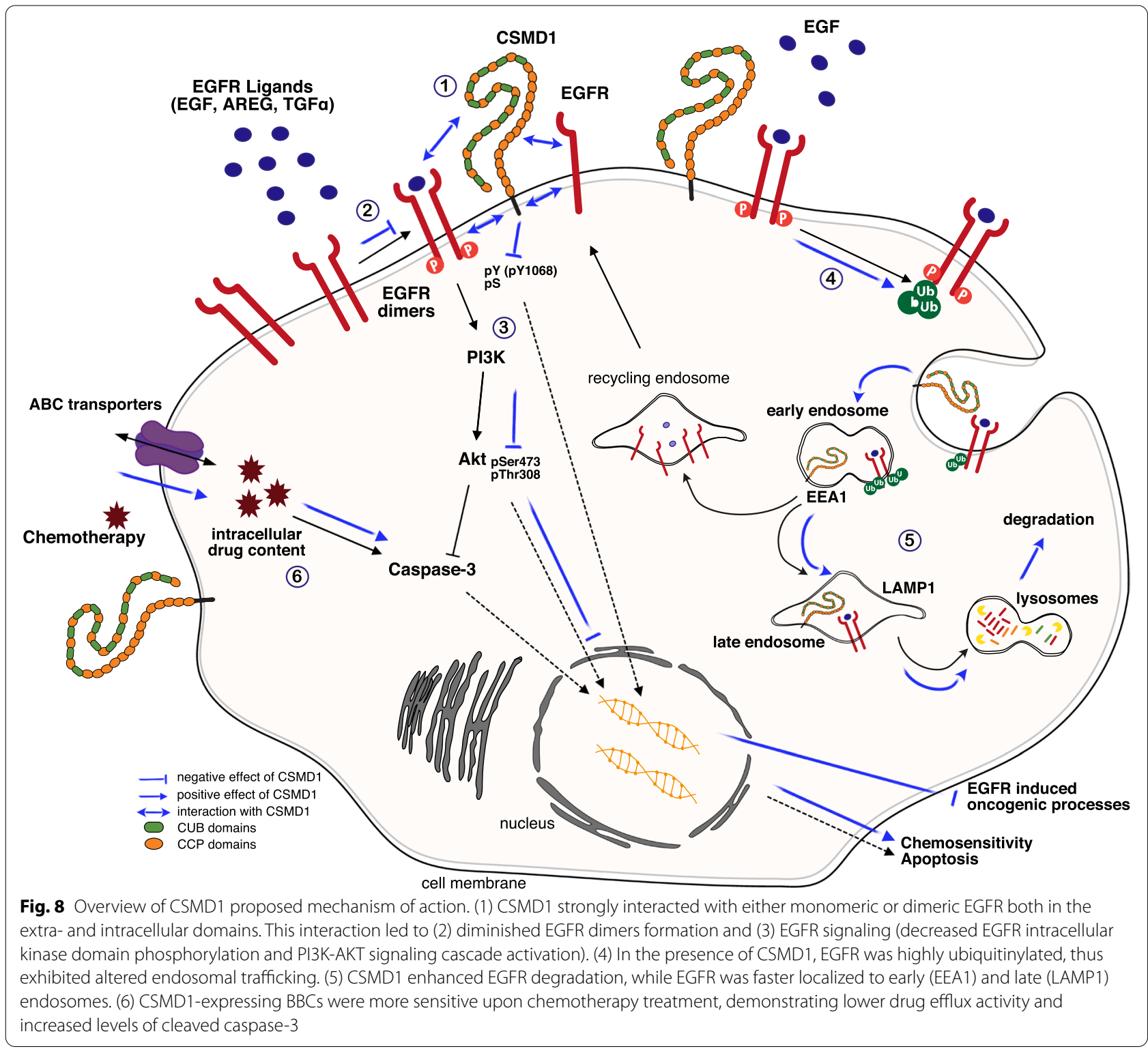

manuscript revisions. However, our in vitro observations were further supported by the clinical data from a realworld RNA sequenced cohort, SCAN-B, where CSDM1 expression showed a trend towards better responses of TNBC patients to adjuvant chemotherapy treatment, while EGFR expression further characterize that response.

\section{Conclusions}

In conclusion, we have characterized one novel mechanism of tumor suppressor activity of CSMD1 and we have identified EGFR as the mediator of this action (Fig. 8). This study highlights the possibility that CSMD1 may be used as a biomarker predicting chemotherapy response in TNBC, which should be also investigated for other subtypes. Thus, CSMD1 expression may help to guide treatment options, aiding a personalized treatment plan. Furthermore, if CSMD1 expression can be upregulated in tumors in vivo, CSMD1 may also be considered as a potential avenue for a novel therapy.

\section{Abbreviations}

AREG: Amphiregulin; BCCs: Breast cancer cells; CCP: Complement control protein; CHX: Cycloheximide; CSMD1: CUB and Sushi multiple domains 1; CTSS: Cathepsin S; EEA1: Early endosome antigen 1; EGFR: Epidermal growth factor receptor; ER: Estrogen receptor; FBS: Fetal bovine serum; GEF: Gefitinib; HER2: Human epidermal growth factor receptor 2; LAMP1: Lysosomal-associated membrane protein 1; LN: Lymph node; NEM: N-Ethylmaleimide; PLA: Proximity ligation assay; TNBC: Triple negative breast cancer; Ub: Ubiquitin. 


\section{Supplementary Information}

The online version contains supplementary material available at https://doi. org/10.1186/s13046-021-02042-1.

Additional file 1: S.Figure 1 Expression of mRNA coding for (A) $E G F$, (B) TGF- $a$ and (C) AREG in MDA-MB-231 CTRL and CSMD1 clonal cells. EGFR gene expression (FPKM) plotted against CSMD1 (FPKM) gene expression in (D) all BC patients and in (E) TNBC patients of SCAN-B cohort (F-G) Protein extracts of MDA-MB-231 BCCs were immunoprecipitated with anti-EGFR. Eluted proteins were analyzed by immunoblotting with (F) anti-phosphotyrosine (pTyr) or anti-EGFR antibody and (G) anti-phosphoserine (pSer) or anti-EGFR antibody, as indicated. (H \& I) Densitometric western blot analysis of total phosphorylation tyrosine and serine residues of EGFR. Bars display mean \pm SD. Mann-Whitney comparison test was used $(*<0.05)$. (J) Binding assays with ${ }^{125}$-labeled EGF in CTRL and CSMD1 MDA-MB-231 BCCs. All experiments were repeated at least 3 times with bars indicating mean \pm SD, grey circles correspond to independent data points for CTRL and CSMD1 groups, respectively. S.Figure 2. (A) Ubiquitinated EGFR was examined via EGFR immunoprecipitation followed by immunoblotting with anti-ubiquitin antibody in denaturing lysates. Representative blots from three independent experiments are presented in CTRL and CSMD1 MDA-MB-231 BCCs. (B) EGFR internalization kinetics using ${ }^{125}$ |-EGF in MDA-MB-231 BCCs. The amounts of internalized and surface 125I-EGF (cpm) where plotted against time upper panel, while the ratio of internalized/surface EGF against time was used to calculate the internalization rate constant ke. (C) Fractionation analysis in cytosol and membrane of CTRL and CSMD1 MDA-MB-231 BCCs upon stimulation with EGF (25 ng/ $\mathrm{mL}$ ) for $2 \mathrm{~h}$. Representative blots are shown. The fractions were blotted for CSMD1, EGFR, EEA1, LAMP1, $\beta$-tubulin and NA/K ATPase (D) Ratio of cytosolic to membrane EGFR was calculated. Bars display mean \pm SD. S. Figure $\mathbf{3}$ Validation of the major findings in BT-20 TNBC cell line (A) Cell lysates were immunoprecipitated using antibodies against CSMD1 or corresponding lg $\mathrm{G}$ control followed by immunodetection of EGFR and CSMD1 in BT-20 cells. (B) Immunoblot analysis of phosphorylated EGFR at the residue Y1068, total EGFR and $\beta$-tubulin used as a loading control. (C) Densitometry of pEGFR Y1068 normalized to total EGFR. (D) Densitometry of pEGFRY1068 normalized to $\beta$-tubulin, and (E) densitometry of total EGFR normalized to $\beta$-tubulin. (F) Immunoblot analysis of phosphorylated Akt at the residue Ser473 (pAkt Ser473), total Akt and GAPDH used as a loading control in BT-20 cells. (G) Densitometry of pAkt Ser473 normalized to total Akt and (H) densitometry of pAkt Ser473 normalized to GAPDH in BT-20 cells. A two-way ANOVA Bonferroni's multiple comparisons test was used when comparing 3 or more groups with 2 variables $\left({ }^{*}<0.05\right)$. (I) EGFR degradation: representative immunoblots of EGFR levels in lysates of BT-20 CTRL and CSMD1 overexpressing cells, pre-treated with translation inhibitor cycloheximide $(100 \mathrm{~g} / \mathrm{mL})$ for $2 \mathrm{~h}$, followed by EGF stimulation $(25 \mathrm{ng} / \mathrm{ml})$ for $0,2,4,8$ hours (h). (J) Quantification of EGFR levels in MDA-MB-231 cells plotted against time. A two-way ANOVA Bonferroni's multiple comparisons test was used when comparing CTRL and CSMD1 groups $\left({ }^{*}<0.05,{ }^{* *}<0.01\right)$. (K) BT-20 CTRL and CSMD1 cells were treated with different chemotherapy agents (doxorubicin and epirubicin) for 48h, and apoptosis was monitored using annexin $V$ staining while live/ dead cell discrimination was performed with Zombie Aqua staining, both using flow cytometry. Bar graphs showing percentages of late apoptotic cells. All experiments were repeated at least 3 times with bars indicating mean $\pm S D$, grey circles correspond to independent data points for CTRL and CSMD1 groups, respectively. A two-way ANOVA Bonferroni's multiple comparisons test was used when comparing CTRL and CSMD1 groups in different concentration of chemotherapy drugs or treatments. S. Figure 4 Validation of the major findings in MDA-MB-468 TNBC cell line (A) Immunoblot analysis of phosphorylated EGFR at the residue Y1068, total EGFR and $\beta$-tubulin in MDA-MB-468 cells. (B) Densitometry of pEGFR-Y1068 normalized to total EGFR. (C) Densitometry of pEGFR-Y1068 normalized to $\beta$-tubulin, and (D) densitometry of total EGFR normalized to $\beta$-tubulin. (E) Immunoblot analysis of phosphorylated Akt at the residue Ser473 (pAkt-Ser473), total Akt and GAPDH in MDA-MB-468 cells. (F) Densitometry of pAkt-Ser473 normalized to total Akt and (G) densitometry of pAkt-Ser473 normalized to GAPDH. A two-way ANOVA Bonferroni's multiple comparisons test was used when comparing 3 or more groups with 2 variables $\left({ }^{*}<0.05\right)$. $(\mathrm{H})$ EGFR degradation in MDA-MB-468 breast cancer cell line: detection of EGFR in lysates of MDA-MB-468 CTRL and CSMD1-overexpressing cells, pre-treated with translation inhibitor cycloheximide $(100 \mathrm{~g} / \mathrm{mL})$ for $2 \mathrm{~h}$ followed by EGF stimulation $(25 \mathrm{ng} / \mathrm{ml})$ for $0,2,4,8$ hours (h). (I) Quantification of EGFR levels in MDA-MB-468 cells plotted against time. A two-way ANOVA Bonferroni's multiple comparisons test was used when comparing CTRL and CSMD1 groups $(*<0.05$, $\left.{ }^{* *}<0.01\right)$. (J) MDA-MB-468 CTRL and CSMD1-overexpressing cells were treated with different chemotherapy agents (doxorubicin and epirubicin) for $48 \mathrm{~h}$, and apoptosis was monitored using annexin $\mathrm{V}$ staining while live/ dead cell discrimination was performed with Zombie Aqua staining, both using flow cytometry. All experiments were repeated at least 3 times with bars indicating mean $\pm S D$, grey circles correspond to independent data points for CTRL and CSMD1 groups, respectively. Bar graphs show percentages of late apoptotic cells. A two-way ANOVA Bonferroni's multiple comparisons test was used when comparing CTRL and CSMD1 groups in different concentration of chemotherapy drugs or treatments. S. Figure 5 (A) Drug efflux activity in MDA-MB-231 CSMD1-overexpressing cells and CTRL cells. Representative histograms are shown. (B) Bar graphs showing gMFI of intracellular doxorubicin content in MDA-MB-231 CSMD1 and CTRL cells. Student's t-test was used when comparing CTRL and CSMD1 (C) Representative immunoblots of autophagy markers p62 and LC3B in lysates of MDA-MB-231 CTRL and CSMD1 cells upon treatment with GEF and CQ and combination of them for $24 \mathrm{~h}$. All experiments were repeated at least 3 times with bars indicating mean \pm SD, grey circles correspond to independent data points for CTRL and CSMD1 groups, respectively. $\mathbf{S}$. Figure 6 Frequency (\%) of CSMD1 expression in (A) all BC patients and in (B) TNBC patients of SCAN-B cohort. (C) Box plot of EGFR gene expression for tumor samples of SCAN-B cohort subset of TNBC stratified according to basal and non-basal like properties. Table S1 List of antibodies used in this study.

\section{Acknowledgements}

Dr. Ben King is kindly acknowledged for critical comments and language correction of this manuscript.

\section{Authors' contributions}

CG, ECT, SG, VP, MM and KSP carried out the experimental work. AMB and CG conceived and designed the study. CG and ECT analyzed the data in the study. JS conducted the bioinformatic analyses of the breast cancer cohort. CG, ECT and $A M B$ wrote the manuscript. All authors have read and approved the final manuscript

\section{Funding}

Open access funding provided by Lund University. This study was supported by Cancerfonden, Royal Physiographic Society in Lund, Längmanska kulturfonden, O.E. and Edla Johanssons Foundation, Malmö Hospital Cancer Foundation, and a grant for clinical research (ALF). The funding bodies had no impact on the design of the study and collection, analysis, and interpretation of data.

\section{Availability of data and materials}

The datasets during and/or analyzed during the current study available from the corresponding author on reasonable request.

\section{Declarations}

Ethics approval and consent to participate

Not applicable for this study.

\section{Consent for publication}

Not applicable for this study.

\section{Competing interests}

The authors declare that they have no competing interests.

\section{Author details}

${ }^{1}$ Department of Translational Medicine, Lund University, Malmö, Sweden.

${ }^{2}$ Experimental Cardiovascular Research Group, Department of Clinical 
Sciences, Lund University, Malmö, Sweden. ${ }^{3}$ Division of Oncology, Department of Clinical Sciences Lund, Lund University, Medicon Village, Lund, Sweden. ${ }^{4}$ Colzyx AB, Lund, Sweden.

Received: 3 February 2021 Accepted: 14 July 2021 Published online: 17 August 2021

\section{References}

1. Escudero-Esparza A, Kalchishkova N, Kurbasic E, Jiang WG, Blom AM. The novel complement inhibitor human CUB and Sushi multiple domains 1 (CSMD1) protein promotes factor I-mediated degradation of C4b and C3b and inhibits the membrane attack complex assembly. FASEB J. 2013;27:5083-93.

2. Kraus DM, Elliott GS, Chute H, Horan T, Pfenninger KH, Sanford SD, Foster $S$, Scully S, Welcher AA, Holers VM. CSMD1 is a novel multiple domain complement-regulatory protein highly expressed in the central nervous system and epithelial tissues. J Immunol. 2006;176:4419-30.

3. Fagerberg L, Hallstrom BM, Oksvold P, Kampf C, Djureinovic D, Odeberg J, Habuka M, Tahmasebpoor S, Danielsson A, Edlund K, et al. Analysis of the human tissue-specific expression by genome-wide integration of transcriptomics and antibody-based proteomics. Mol Cell Proteomics. 2014;13:397-406.

4. Lau WL, Scholnick SB. Identification of two new members of the CSMD gene family. Genomics. 2003;82:412-5.

5. Kamal M, Shaaban AM, Zhang L, Walker C, Gray S, Thakker N, Toomes C, Speirs V, Bell SM. Loss of CSMD1 expression is associated with high tumour grade and poor survival in invasive ductal breast carcinoma. Breast Cancer Res Treat. 2010;121:555-63.

6. Sun PC, Uppaluri R, Schmidt AP, Pashia ME, Quant EC, Sunwoo JB, Gollin SM, Scholnick SB. Transcript map of the 8p23 putative tumor suppressor region. Genomics. 2001;75:17-25.

7. Liu Y, Fu X, Tang Z, Li C, Xu Y, Zhang F, Zhou D, Zhu C. Altered expression of the CSMD1 gene in the peripheral blood of schizophrenia patients. BMC Psychiatry. 2019;19:113.

8. Steen VM, Nepal C, Ersland KM, Holdhus R, Naevdal M, Ratvik SM, Skrede S, Havik B. Neuropsychological deficits in mice depleted of the schizophrenia susceptibility gene CSMD1. PLoS One. 2013;8:e79501.

9. Lee AS, Rusch J, Lima AC, Usmani A, Huang N, Lepamets M, Vigh-Conrad KA, Worthington RE, Magi R, Wu X, et al. Rare mutations in the complement regulatory gene CSMD1 are associated with male and female infertility. Nat Commun. 2019;10:4626.

10. Liu P, Morrison C, Wang L, Xiong D, Vedell P, Cui P, Hua X, Ding F, Lu Y, James $\mathrm{M}$, et al. Identification of somatic mutations in non-small cell lung carcinomas using whole-exome sequencing. Carcinogenesis. 2012;33:1270-6.

11. Zhang R, Song C. Loss of CSMD1 or 2 may contribute to the poor prognosis of colorectal cancer patients. Tumour Biol. 2014;35:4419-23.

12. Nilsson SC, Trouw LA, Renault N, Miteva MA, Genel F, Zelazko M, Marquart H, Muller K, Sjoholm AG, Truedsson L, et al. Genetic, molecular and functional analyses of complement factor I deficiency. Eur J Immunol. 2009:39:310-23.

13. Scholnick SB, Richter TM. The role of CSMD1 in head and neck carcinogenesis. Genes Chromosomes Cancer. 2003;38:281-3.

14. Toomes C, Jackson A, Maguire K, Wood J, Gollin S, Ishwad C, Paterson I, Prime S, Parkinson K, Bell S, et al. The presence of multiple regions of homozygous deletion at the CSMD1 locus in oral squamous cell carcinoma question the role of CSMD1 in head and neck carcinogenesis. Genes Chromosomes Cancer. 2003;37:132-40.

15. Midorikawa Y, Yamamoto S, Tsuji S, Kamimura N, Ishikawa S, Igarashi $H_{\text {, }}$ Makuuchi M, Kokudo N, Sugimura H, Aburatani H. Allelic imbalances and homozygous deletion on 8p23.2 for stepwise progression of hepatocarcinogenesis. Hepatology. 2009;49:513-22.

16. Richter TM, Tong BD, Scholnick SB. Epigenetic inactivation and aberrant transcription of CSMD1 in squamous cell carcinoma cell lines. Cancer Cell Int. 2005;5:29.

17. Scholnick SB, Haughey BH, Sunwoo JB. el-Mofty SK, Baty JD, Piccirillo JF, Zequeira MR: Chromosome 8 allelic loss and the outcome of patients with squamous cell carcinoma of the supraglottic larynx. J Natl Cancer Inst. 1996;88:1676-82.
18. Escudero-Esparza A, Bartoschek M, Gialeli C, Okroj M, Owen S, Jirstrom K, Orimo A, Jiang WG, Pietras K, Blom AM. Complement inhibitor CSMD1 acts as tumor suppressor in human breast cancer. Oncotarget. 2016;7:76920-33.

19. Tang MR, Wang YX, Guo S, Han SY, Wang D. CSMD1 exhibits antitumor activity in A375 melanoma cells through activation of the Smad pathway. Apoptosis. 2012;17:927-37.

20. Lang MF, Yang S, Zhao C, Sun G, Murai K, Wu X, Wang J, Gao H, Brown CE, Liu X, et al. Genome-wide profiling identified a set of miRNAs that are differentially expressed in glioblastoma stem cells and normal neural stem cells. PLoS One. 2012;7:e36248.

21. Zhu Q, Gong L, Wang J, Tu Q, Yao L, Zhang JR, Han XJ, Zhu SJ, Wang SM, Li YH, Zhang W. miR-10b exerts oncogenic activity in human hepatocellular carcinoma cells by targeting expression of CUB and sushi multiple domains 1 (CSMD1). BMC Cancer. 2016;16:806.

22. Kamal M, Holliday DL, Morrison EE, Speirs V, Toomes C, Bell SM. Loss of CSMD1 expression disrupts mammary duct formation while enhancing proliferation, migration and invasion. Oncol Rep. 2017;38:283-92.

23. Gialeli C, Gungor B, Blom AM. Novel potential inhibitors of complement system and their roles in complement regulation and beyond. Mol Immunol. 2018;102:73-83.

24. Abdillahi SM, Maass T, Kasetty G, Stromstedt AA, Baumgarten M, Tati R, Nordin SL, Walse B, Wagener R, Schmidtchen A, Morgelin M. Collagen VI Contains Multiple Host Defense Peptides with Potent In Vivo Activity. J Immunol. 2018;201:1007-20.

25. Hsu SC, Hung MC. Characterization of a novel tripartite nuclear localization sequence in the EGFR family. J Biol Chem. 2007;282:10432-40.

26. Bjorkelund H, Gedda L, Barta P, Malmqvist M, Andersson K. Gefitinib induces epidermal growth factor receptor dimers which alters the interaction characteristics with (1)(2)(5)I-EGF. PLoS One. 2011;6:e24739.

27. Vallon-Christersson J, Hakkinen J, Hegardt C, Saal LH, Larsson C, Ehinger A, Lindman H, Olofsson H, Sjoblom T, Warnberg F, et al. Cross comparison and prognostic assessment of breast cancer multigene signatures in a large population-based contemporary clinical series. Sci Rep. 2019:9:12184.

28. Escudero-Esparza A, Bartoschek M, Gialeli C, Okroj M, Owen S, Jirstrom K, Orimo A, Jiang WG, Pietras K, Blom AM. Complement inhibitor CSMD1 acts as tumor suppressor in human breast cancer. Oncotarget. 2016;7(47):76920-33.

29. Wilson KJ, Gilmore JL, Foley J, Lemmon MA, Riese DJ 2nd. Functional selectivity of EGF family peptide growth factors: implications for cancer. Pharmacol Ther. 2009;122:1-8.

30. Jones JT, Akita RW, Sliwkowski MX. Binding specificities and affinities of egf domains for ErbB receptors. FEBS Lett. 1999;447:227-31.

31. Tomas A, Futter CE, Eden ER. EGF receptor trafficking: consequences for signaling and cancer. Trends Cell Biol. 2014;24:26-34.

32. Kitaeva KV, Rutland CS, Rizvanov AA, Solovyeva W. Cell culture based in vitro test systems for anticancer drug screening. Front Bioeng Biotechnol. 2020;8:322

33. Rimawi MF, Shetty PB, Weiss HL, Schiff R, Osborne CK, Chamness GC, Elledge RM. Epidermal growth factor receptor expression in breast cancer association with biologic phenotype and clinical outcomes. Cancer. 2010;116:1234-42.

34. Zhang M, Zhang X, Zhao S, Wang Y, Di W, Zhao G, Yang M, Zhang Q. Prognostic value of survivin and EGFR protein expression in triple-negative breast cancer (TNBC) patients. Target Oncol. 2014;9:349-57.

35. Rao C, Shetty J, Prasad KH. Immunohistochemical profile and morphology in triple - negative breast cancers. J Clin Diagn Res. 2013;7:1361-5.

36. Gupta GK, Collier AL, Lee D, Hoefer RA, Zheleva V, Siewertsz van Reesema LL, Tang-Tan AM, Guye ML, Chang DZ, Winston JS, et a. Perspectives on triple-negative breast cancer: current treatment strategies, unmet needs, and potential targets for future therapies. Cancers (Basel). 2020;12(9):2392.

37. Lev S. Targeted therapy and drug resistance in triple-negative breast cancer: the EGFR axis. Biochem Soc Trans. 2020;48:657-65.

38. Burgess SJ, Gibbs H, Toomes C, Coletta PL, Bell SM. The role of Csmd1 during mammary gland development. Genes (Basel). 2021;12(2):62.

39. McGough AM, Staiger CJ, Min JK, Simonetti KD. The gelsolin family of actin regulatory proteins: modular structures, versatile functions. FEBS Lett. 2003;552:75-81. 
40. Gostner JM, Fong D, Wrulich OA, Lehne F, Zitt M, Hermann M, Krobitsch S, Martowicz A, Gastl G, Spizzo G. Effects of EpCAM overexpression on human breast cancer cell lines. BMC Cancer. 2011;11:45.

41. Wilkinson RDA, Burden RE, McDowell SH, McArt DG, McQuaid S, Bingham V, Williams R, Cox OT, O'Connor R, McCabe N, et al. A novel role for Cathepsin $\mathrm{S}$ as a potential biomarker in triple negative breast cancer. J Oncol. 2019;2019:3980273.

42. Gautam J, Banskota S, Lee H, Lee YJ, Jeon YH, Kim JA, Jeong BS. Downregulation of cathepsin $\mathrm{S}$ and matrix metalloproteinase-9 via Src, a nonreceptor tyrosine kinase, suppresses triple-negative breast cancer growth and metastasis. Exp Mol Med. 2018;50:118.

43. Conte A, Sigismund S. Chapter Six - The Ubiquitin network in the control of EGFR endocytosis and signaling. Prog Mol Biol Transl Sci. 2016;141:225-76.

44. Ruicci KM, Meens J, Sun RX, Rizzo G, Pinto N, Yoo J, Fung K, MacNeil D, Mymryk JS, Barrett JW, et al. A controlled trial of HNSCC patient-derived xenografts reveals broad efficacy of PI3Kalpha inhibition in controlling tumor growth. Int J Cancer. 2019;145:2100-6.

45. Chavez KJ, Garimella SV, Lipkowitz S. Triple negative breast cancer cell lines: one tool in the search for better treatment of triple negative breast cancer. Breast Dis. 2010;32:35-48.

46. Andre F, Ciruelos EM, Juric D, Loibl S, Campone M, Mayer IA, Rubovszky G, Yamashita T, Kaufman B, Lu YS, et al. Alpelisib plus fulvestrant for PIK3CAmutated, hormone receptor-positive, human epidermal growth factor receptor-2-negative advanced breast cancer: final overall survival results from SOLAR-1. Ann Oncol. 2021;32:208-17.

47. Sigismund S, Avanzato D, Lanzetti L. Emerging functions of the EGFR in cancer. Mol Oncol. 2018;12:3-20.

48. Longva KE, Blystad FD, Stang E, Larsen AM, Johannessen LE, Madshus IH. Ubiquitination and proteasomal activity is required for transport of the EGF receptor to inner membranes of multivesicular bodies. J Cell Biol. 2002;156:843-54.

49. Cleator S, Parton M, Dowsett M. The biology of neoadjuvant chemotherapy for breast cancer. Endocr Relat Cancer. 2002;9:183-95.

50. Wang F, Li D, Zheng Z, Kin Wah To K, Chen Z, Zhong M, Su X, Chen L, Fu L. Reversal of ABCB1-related multidrug resistance by ERK5-IN-1. J Exp Clin Cancer Res. 2020;39:50.

51. Aldonza MBD, Reyes RDD, Kim YS, Ku J, Barsallo AM, Hong JY, Lee SK, Ryu HS, Park Y, Cho JY, Kim Y. Chemotherapy confers a conserved secondary tolerance to EGFR inhibition via AXL-mediated signaling bypass. Sci Rep. $2021 ; 11: 8016$

\section{Publisher's Note}

Springer Nature remains neutral with regard to jurisdictional claims in published maps and institutional affiliations.
Ready to submit your research? Choose BMC and benefit from:

- fast, convenient online submission

- thorough peer review by experienced researchers in your field

- rapid publication on acceptance

- support for research data, including large and complex data types

- gold Open Access which fosters wider collaboration and increased citations

- maximum visibility for your research: over 100M website views per year

At BMC, research is always in progress.

Learn more biomedcentral.com/submissions 